\title{
POTENTIAL ESTIMATES IN PARABOLIC OBSTACLE PROBLEMS
}

\author{
Christoph Scheven \\ Universität Erlangen-Nürnberg, Department Mathematik \\ Cauerstraße 11, 91058 Erlangen, Germany; scheven@mi.uni-erlangen.de
}

\begin{abstract}
For parabolic obstacle problems with quadratic growth, we give pointwise estimates both for the solutions and their gradients in terms of potentials of the given data. As applications, we derive Lorentz space estimates if the data satisfies the corresponding Lorentz space regularity. Moreover, we discuss a borderline case in the regularity theory, the question of boundedness and continuity of the gradients as well as of the solutions itself.
\end{abstract}

\section{Introduction and summary of results}

In the present work, we establish pointwise estimates by potentials for solutions to parabolic obstacle problems. Obstacle problems play a prominent role in various applications, for example in mechanics or control theory, cf. [4, 22], but also in other fields of mathematics such as potential theory, where solutions to obstacle problems prove useful as approximations of super-solutions [16, 18, 23].

Here, we treat obstacle problems that are related to equations of the type

$$
\partial_{t} u-\operatorname{div} a(x, t, D u)=f \quad \text { on } \Omega_{T}:=\Omega \times(0, T)
$$

for a vector-field $a: \Omega_{T} \times \mathbf{R}^{n} \rightarrow \mathbf{R}^{n}$ with so-called quadratic growth (see Section 1.2 for the precise assumptions), where $\Omega \subset \mathbf{R}^{n}$ is a bounded domain of dimension $n \geq 2$ and $f \in L^{2}\left(\Omega_{T}\right)$. Additionally, we impose an obstacle constraint of the form $u \geq \psi$ a.e. on $\Omega_{T}$ for an obstacle function $\psi: \Omega_{T} \rightarrow \mathbf{R}$. Our general assumptions on the obstacle function are $\psi \in L^{2}\left(0, T ; W^{1,2}(\Omega)\right)$ and $\partial_{t} \psi \in L^{2}\left(\Omega_{T}\right)$ for the zero order estimates, and for the gradient estimates additionally $\psi \in L^{2}\left(0, T ; W^{2,2}(\Omega)\right)$. Some of our results require even stronger regularity properties of the obstacle, which will be stated seperately for the particular cases. At no point, however, we assume monotonicity of the obstacle with respect to time.

1.1. A brief history of the problem. In order to illustrate the kind of estimates that we have in mind, we consider the easiest case of the linear Poisson equation

$$
-\Delta u=f \quad \text { on } \mathbf{R}^{n}
$$

for a given function $f \in L^{2}\left(\mathbf{R}^{n}\right)$. The classical representation formula for the solution yields pointwise estimates of the type

$$
|u(x)| \leq c \int_{\mathbf{R}^{n}} \frac{|f(y)|}{|x-y|^{n-2}} d y \quad \text { and } \quad|D u(x)| \leq c \int_{\mathbf{R}^{n}} \frac{|f(y)|}{|x-y|^{n-1}} d y
$$

doi:10.5186/aasfm.2012.3730

2010 Mathematics Subject Classification: Primary 35K86, 35B65, 31B35.

Key words: Parabolic obstacle problems, regularity of solutions, non-linear potential theory. 
for a.e. $x \in \mathbf{R}^{n}$. In other words, the solution and its gradient can be estimated pointwise in terms of the classical Riesz potential $I_{2}(f)$, respectively $I_{1}(f)$. In their seminal work [20], Kilpeläinen and Malý were able to give analogous estimates for solutions to non-linear elliptic equations of $p$-Laplace type with $p \neq 2$. More precisely, they considered non-negative $p$-superharmonic functions $u$ with $-\Delta_{p} u=f \geq 0$ on a ball $B_{R}\left(x_{0}\right)$, for which they established a bound of the type

$$
u\left(x_{0}\right) \leq c\left(f_{B_{R / 2}\left(x_{0}\right)} u^{\gamma} d x\right)^{\frac{1}{\gamma}}+c \mathbf{W}_{1, p}^{f}\left(x_{0}, R\right)
$$

for some $\gamma>p-1$ and the non-linear Wolff-potential

$$
\mathbf{W}_{\beta, p}^{f}\left(x_{0}, R\right):=\int_{0}^{R}\left[\frac{|f|\left(B_{\varrho}\left(x_{0}\right)\right)}{\varrho^{n-\beta p}}\right]^{\frac{1}{p-1}} \frac{d \varrho}{\varrho}
$$

for any $\beta \in(0, n]$, where we used the notation $|f|\left(B_{\varrho}\left(x_{0}\right)\right):=\int_{B_{\varrho}\left(x_{0}\right)}|f| d x$. We note that in the case $p=2$, the Wolff-potential is a localized version of the Riesz potential $I_{2 \beta}(f)$, which makes the estimate (1.3) a natural extension of the Riesz potential estimate (1.2) from the linear case. In the case $p \neq 2$, the Wolff-potentials can be estimated by iterated Riesz potentials of Havin-Maz'ya type, which makes classical Riesz potential estimates applicable to bound the right-hand side of (1.3) further. Results related to (1.3) have also been established by Kilpeläinen and Malý [19] and Kilpeläinen and Zhong [21]. Trudinger and Wang [36], using a different approach, moreover extended the above mentioned result to a very general setting. However, the question of an analogous estimate for the gradient, i.e. the generalization of the gradient estimate in (1.2) to non-linear equations, remained open until it was recently settled by Mingione [28] for the case $p=2$ and later by Duzaar and Mingione $[10,11,12,13]$ in the general case. For solutions to elliptic equations related to the model case

$$
-\operatorname{div}\left(a(x)|D u|^{p-2} D u\right)=f \quad \text { on } B_{R}\left(x_{0}\right),
$$

with Dini-continuous coefficients $a: \Omega \rightarrow \mathbf{R}$, they gave an estimate of the form

$$
\left|D u\left(x_{0}\right)\right| \leq c f_{B_{R / 2}\left(x_{0}\right)}|D u| d x+c \mathbf{W}_{\frac{1}{p}, p}^{f}\left(x_{0}, R\right) .
$$

Another salient conclusion of the approach by Duzaar and Mingione [11] is a borderline regularity result situated between classical Calderón-Zygmund- and $C^{1, \alpha_{-}}$ estimates, which was so far not accessible by previous techniques. Namely, for solutions to (1.4), Duzaar and Mingione were able to establish continuity of the gradient, provided the right-hand side $f$ is contained in the Lorentz space $L\left(n, \frac{1}{p-1}\right)$. This result is sharp at least in the case $p=2$, in the sense that $f \in L(n, q)$ for any $q>1$ is not sufficient to deduce even boundedness of the gradient, as observed by Cianchi in the case of the Poisson equation [7]. Furthermore, the result is sharp with respect to the regularity of the coefficient function $a$ by a striking counter example by Jin, Maz'ya and Van Schaftingen [17], who constructed a solution with unbounded gradient to a linear homogeneous equation with continuous, but not Dini-continuous coefficients.

Concerning gradient potential estimates in the parabolic setting, the results by Duzaar and Mingione [10] are restricted to problems with quadratic growth, i.e. with 
$p=2$. This is due to the anisotropic scaling behaviour of the parabolic equations that makes it necessary to work with so-called intrinsic cylinders, following the fundamental idea of intrinsic geometry by DiBenedetto [9]. These intrinsic cylinders depend on the solution and may in particular degenerate if $|D u|$ becomes large. The appearance of possibly degenerate cylinders causes intricate problems for the definition of suitable parabolic versions of the Wolff potentials in (1.5). The parabolic setting with $p \neq 2$ therefore poses challenging problems that are to the knowledge of the author open even in the obstacle-free case. Therefore, we restrict ourselves to the case $p=2$ in the present work. The general case $p \neq 2$ in the elliptic setting will be addressed in the forthcoming works [33, 32].

While the present work investigates properties of the spatial derivative or the solution itself, the regularity of the time derivative has been addressed in a recent interesting work by Lindqvist [25]. More precisely, he established $\partial_{t} u \in L_{\mathrm{loc}}^{p /(p-1)}\left(\Omega_{T}\right)$ for the solution $u$ of an obstacle problem associated to the evolutionary $p$-Laplace equation.

1.2. Assumptions and formulation of the problem. Before we state our results more precisely, we specify our assumptions. We assume that $a: \Omega_{T} \times \mathbf{R}^{n} \rightarrow \mathbf{R}^{n}$ is a Carathéodory function, i.e. $\mathbf{R}^{n} \ni \xi \mapsto a(z, \xi)$ is continuous for a.e. $z \in \Omega_{T}$ and $\Omega_{T} \ni z \mapsto a(z, \xi)$ is measurable for every $\xi \in \mathbf{R}^{n}$. For given constants $0<\nu \leq 1 \leq L$, we impose the ellipticity and growth properties

$$
\begin{aligned}
\left(a(z, \xi)-a\left(z, \xi_{0}\right)\right) \cdot\left(\xi-\xi_{0}\right) & \geq \nu\left|\xi-\xi_{0}\right|^{2}, \\
|a(z, \xi)| & \leq L(1+|\xi|),
\end{aligned}
$$

for all $z \in \Omega_{T}$ and $\xi, \xi_{0} \in \mathbf{R}^{n}$. For the gradient estimates, we will assume moreover that $\xi \mapsto a(z, \xi)$ is differentiable for a.e. $z \in \Omega_{T}$ and satisfies the corresponding ellipticity and growth estimates on the level of the derivative $D_{\xi} a$, more precisely

$$
\begin{gathered}
D_{\xi} a(z, \xi) \zeta \cdot \zeta \geq \nu|\zeta|^{2}, \\
\left|D_{\xi} a(z, \xi)\right| \leq L,
\end{gathered}
$$

for all $z \in \Omega_{T}$ and $\xi, \zeta \in \mathbf{R}^{n}$. We point out that, if $D_{\xi} a$ exists, the ellipticity condition (1.8) implies (1.6), possibly with a smaller constant $\tilde{\nu}=\frac{\nu}{c}$ instead of $\nu$. Moreover, for the gradient estimates we assume that $\Omega \ni x \mapsto a(x, t, \xi)$ is Dini-continuous in the sense that

$$
\left|a(x, t, \xi)-a\left(x_{0}, t, \xi\right)\right| \leq 2 L \omega\left(\left|x-x_{0}\right|\right)(1+|\xi|)
$$

holds for all $x, x_{0} \in \Omega, t \in(0, T)$ and $\xi \in \mathbf{R}^{n}$, where $\omega:[0, \infty) \rightarrow[0,1]$ is a nondecreasing modulus of continuity that satisfies the Dini-condition

$$
\int_{0}^{R} \omega(\varrho) \frac{d \varrho}{\varrho}<\infty
$$

for all $R>0$. For the regularity of the partial map $(0, T) \ni t \mapsto a(x, t, \xi)$ however, we do not need to impose anything except measurability.

As mentioned above, we consider an obstacle function $\psi \in L^{2}\left(0, T ; W^{1,2}(\Omega)\right)$ with $\partial_{t} \psi \in L^{2}\left(\Omega_{T}\right)$, and for the gradient potential estimates we assume additionally $\psi \in$ $L^{2}\left(0, T ; W^{2,2}(\Omega)\right)$. We note that some of our results require even stronger regularity properties of the obstacle, as indicated below. Following [31], we consider localizable solutions to obstacle problems that solve a variational inequality on every sufficiently 
regular subset $\mathcal{O}_{I}:=\mathcal{O} \times\left(t_{1}, t_{2}\right) \subset \Omega_{T}$. The solution spaces adapted to the concept of localizable solutions are defined as

$$
K\left(\psi, g ; \mathcal{O}_{I}\right):=\left\{u \in C^{0}\left(\left[t_{1}, t_{2}\right] ; L^{2}(\mathcal{O})\right) \cap\left[g+L^{2}\left(t_{1}, t_{2} ; W_{0}^{1,2}(\mathcal{O})\right)\right]: u \geq \psi \text { a.e. on } \mathcal{O}_{I}\right\},
$$

for given boundary values $g \in L^{2}\left(0, T ; W^{1,2}(\Omega)\right)$ and any subset $\mathcal{O}_{I}:=\mathcal{O} \times\left(t_{1}, t_{2}\right) \subset$ $\Omega_{T}$. For the space of admissible comparison maps however, we have to restrict ourselves to the smaller space

$$
K^{\prime}\left(\psi, g ; \mathcal{O}_{I}\right):=\left\{v \in K\left(\psi, g ; \mathcal{O}_{I}\right): \partial_{t} v \in L^{2}\left(t_{1}, t_{2} ; W^{-1,2}(\mathcal{O})\right)\right\} .
$$

The restriction to comparison maps with a distributional time derivative in $L^{2}\left(t_{1}, t_{2}\right.$; $\left.W^{1,2}(\mathcal{O})\right)$ is necessary for writing down the weak formulation of the obstacle problem. Namely, for a solution $u$, we require $u \in K\left(\psi, g ; \Omega_{T}\right)$, together with the variational inequality

$$
\begin{aligned}
& \int_{0}^{T}\left\langle\partial_{t} v, v-u\right\rangle d t+\int_{\Omega_{T}} a(z, D u) \cdot D(v-u) d z+\frac{1}{2}\|(v-u)(\cdot, 0)\|_{L^{2}}^{2} \\
& \geq \int_{\Omega_{T}} F \cdot D(v-u)+f(v-u) d z
\end{aligned}
$$

for every comparison function $v \in K^{\prime}\left(\psi, g ; \mathcal{O}_{I}\right)$. Here, $F \in L^{2}\left(\Omega_{T}, \mathbf{R}^{n}\right)$ and $f \in$ $L^{2}\left(\Omega_{T}\right)$ are given. From the discussion in [31] however, it becomes clear that the above formulation is not the most natural one for the analysis of regularity properties since it is not of local nature. Therefore, we employ the following localizable notion of a solution to the obstacle problem (1.12) that was introduced in [31] (see also [30]).

Definition 1.1. A map $u \in K\left(\psi, g ; \Omega_{T}\right)$ is called a localizable solution of the obstacle problem (1.12) if for every subdomain $\mathcal{O}_{I}:=\mathcal{O} \times\left(t_{1}, t_{2}\right) \subset \Omega_{T}$, where $\mathcal{O}=\widetilde{\mathcal{O}} \cap \Omega$ with a Lipschitz regular domain $\widetilde{\mathcal{O}} \subset \mathbf{R}^{n}$ and $I=\left(t_{1}, t_{2}\right) \subset \mathbf{R}$, the following two conditions hold.

(i) The map $u$ satisfies the extension property $K^{\prime}\left(\psi, u ; \mathcal{O}_{I}\right) \neq \varnothing$;

(ii) for all comparison maps $v \in K^{\prime}\left(\psi, u ; \mathcal{O}_{I}\right)$, the map $u$ satisfies the variational inequality

$$
\begin{aligned}
& \int_{t_{1}}^{t_{2}}\left\langle\partial_{t} v, v-u\right\rangle_{\mathcal{O}} d t+\int_{\mathcal{O}_{I}} a(z, D u) \cdot D(v-u) d z+\frac{1}{2}\left\|(v-u)\left(\cdot, t_{1}\right)\right\|_{L^{2}(\mathcal{O})}^{2} \\
& \geq \int_{\mathcal{O}_{I}} F \cdot D(v-u)+f(v-u) d z
\end{aligned}
$$

where $\langle\cdot, \cdot\rangle_{\mathcal{O}}$ denotes the dual pairing between $W^{-1,2}(\mathcal{O})$ and $W_{0}^{1,2}(\mathcal{O})$.

For existence results for localizable solutions we refer to [31]. We note that the techniques in the mentioned work yield existence for obstacles that admit a distributional time derivative $\partial_{t} u \in L^{2}\left(0, T ; W^{-1,2}(\Omega)\right)$. For existence results for more irregular obstacles we refer to [26].

Now we are in a position to state our results.

1.3. Gradient potential estimates. For the gradient estimates, we consider localizable solutions $u \in K\left(\psi, g ; \Omega_{T}\right)$-in the sense of Definition 1.1 - of the variational inequality (1.12) without divergence term, i.e. with $F=0$. We impose the assumptions listed above, in particular we consider obstacles satisfying 
$\psi \in L^{2}\left(0, T ; W^{2,2}(\Omega)\right)$ and $\partial_{t} \psi \in L^{2}\left(\Omega_{T}\right)$. In this situation, we derive a gradient potential estimate of the form

$$
\begin{aligned}
\left|D u\left(z_{0}\right)\right| \leq & 1+c\left(f_{C_{R}\left(z_{0}\right)}|D u|^{2} d z\right)^{\frac{1}{2}}+c \mathbf{P}_{1}^{\Psi}\left(z_{0}, R\right) \\
& +c \int_{0}^{R} \omega(\varrho)\left[\frac{|D \psi|^{2}\left(C_{\varrho}\left(z_{0}\right)\right)}{\varrho^{n+2}}\right]^{\frac{1}{2}} \frac{d \varrho}{\varrho}
\end{aligned}
$$

for every Lebesgue point $z_{0} \in \Omega_{T}$ of $D u$ and a sufficiently small radius $R>0$, where the right-hand side potential is defined by

$$
\mathbf{P}_{1}^{\Psi}\left(z_{0}, R\right):=\int_{0}^{R}\left[\frac{\Psi^{2}\left(C_{\varrho}\left(z_{0}\right)\right)}{\varrho^{n}}\right]^{\frac{1}{2}} \frac{d \varrho}{\varrho}, \quad \text { for } \Psi:=|f|+\left|\partial_{t} \psi\right|+\left|D^{2} \psi\right| .
$$

Here, we used the short-hand notation $\Psi^{2}\left(C_{\varrho}\left(z_{0}\right)\right):=\int_{C_{\varrho}\left(z_{0}\right)} \Psi^{2} d z$. Clearly, the last term in (1.14) can be omitted if $a(x, t, \xi) \equiv \tilde{a}(t, \xi)$ is independent from the spatial variable, since in this case, the assumptions are satisfied with $\omega \equiv 0$. For the proof of (1.14) we refer to Theorem 4.1. The main idea of the proof, which goes back to Mingione [28], is to compare the given solutions with solutions to less difficult problems, to which classical regularity theory applies. In the present situation, we need to implement a comparison argument consisting of three steps. In a first step, we reduce the problem to a parabolic equation, i.e. without an obstacle, then to a homogeneous parabolic equation and in the last step to a parabolic equation with frozen coefficients. The solution of the latter is of class $C_{\text {loc }}^{1, \beta}$ for some $\beta \in(0,1)$ and satisfies corresponding excess decay estimates, which we carry over to the solution of the obstacle problem by comparison estimates. The resulting excess decay estimates can then be iterated to yield the potential estimate (1.14) for the spatial gradient.

The potential $\mathbf{P}_{1}^{\Psi}$ can be estimated by iterated Riesz potentials of Havin-Maz'ya type, cf. (6.6). Consequently, classical Riesz potential estimates in various scales of function spaces are applicable to the right-hand side of (1.14) and yield corresponding estimates for the spatial gradient of solutions to obstacle problems. As an example, we present the resulting Lorentz space estimates for the gradient of solutions. For any $2<r<N:=n+2$ and $1 \leq s \leq \infty$, we prove that

$$
f, \partial_{t} \psi,\left|D^{2} \psi\right| \in L(r, s) \quad \text { and } \quad|D \psi| \in L\left(\frac{N r}{N-r}, s\right) \text { implies }|D u| \in L_{\mathrm{loc}}\left(\frac{N r}{N-r}, s\right),
$$

and we have the borderline result that

$$
f, \partial_{t} \psi,\left|D^{2} \psi\right| \in L^{2}(\Omega) \quad \text { and } \quad|D \psi| \in L\left(\frac{2 N}{N-2}, \infty\right) \quad \text { implies } \quad|D u| \in L_{\mathrm{loc}}\left(\frac{2 N}{N-2}, \infty\right) .
$$

For the above implications, we also prove the corresponding local estimates, cf. Corollary 4.3. These results can be seen as a refinement of the Calderón-Zygmund estimates for parabolic obstacle problems presented in [5,31], which in turn are based on the theory developed in $[1,14,29]$ for obstacle-free problems. More precisely, the cited works establish estimates in Lebesgue spaces under weaker assumptions on the obstacle and the structure function $a$, while here, we give the corresponding results on the finer Lorentz scale in the case $p=2$ and for more regular obstacles and structure functions.

Clearly, the potential estimate (1.14) further yields a criterion under which solutions have locally bounded gradients, namely if the right-hand side is bounded independently from $z_{0} \in \mathcal{O}_{I}$ for every $\mathcal{O}_{I} \Subset \Omega$. If more strongly, the right-hand side 
of (1.14) vanishes in the limit $R \searrow 0$, uniformly in $z_{0} \in \mathcal{O}_{I}$, we can employ the excess decay estimates mentioned above in order to establish even continuity of the spatial gradient. More precisely, the condition

$$
\left.\begin{array}{c}
\lim _{R \searrow 0} \sup _{\mathcal{O}_{I}} \mathbf{P}_{1}^{\Psi}(\cdot, R)=0 \\
\lim _{R \searrow 0} \sup _{\mathcal{O}_{I}} \int_{0}^{R} \omega(\varrho)\left[f_{C_{\varrho}\left(z_{0}\right)}|D \psi|^{2} d z\right]^{\frac{1}{2}} \frac{d \varrho}{\varrho}=0
\end{array}\right\} \text { for all } \mathcal{O}_{I} \Subset \Omega_{T}
$$

implies $D u \in C_{\text {loc }}^{0}\left(\Omega_{T}, \mathbf{R}^{n}\right)$, see Theorem 5.2. The preceding condition on the potentials holds true e.g. if the data is contained in certain Lorentz spaces. This yields the following Lorentz space criterion for $C^{1}$-regularity (cf. Corollary 5.3).

(1.16) $f, \partial_{t} \psi,\left|D^{2} \psi\right| \in L(n+2,1)$ and $|D \psi| \in L_{\mathrm{loc}}^{\infty}\left(\Omega_{T}\right)$ implies $D u \in C_{\mathrm{loc}}^{0}\left(\Omega_{T}, \mathbf{R}^{n}\right)$.

In the case of a vector field $a(x, t, \xi)=a(t, \xi)$ without $x$-dependence, the assumption $|D \psi| \in L_{\mathrm{loc}}^{\infty}\left(\Omega_{T}\right)$ can be omitted.

Finally, we mention the following criterion for $C^{1, \alpha}$-regularity of solutions, see Lemma 5.4. For this result we assume that $x \mapsto a(x, t, \xi)$ is Hölder continuous, that there holds $|D \psi| \in L_{\mathrm{loc}}^{\infty}\left(\Omega_{T}\right)$ and that we have a Morrey-type condition

$$
\sup _{z_{0} \in \mathcal{O}_{I}} \sup _{0<\varrho<1} \varrho^{2-2 \gamma} f_{C_{\varrho}\left(z_{0}\right) \cap \Omega_{T}}|f|^{2}+\left|\partial_{t} \psi\right|^{2}+\left|D^{2} \psi\right|^{2} d z<\infty
$$

for every subset $\mathcal{O}_{I} \Subset \Omega_{T}$ and some $\gamma>0$. Under these assumptions, we prove $u \in C_{\text {loc }}^{1, \alpha}\left(\Omega_{T}\right)$ for some exponent $\alpha \in(0,1)$ depending on the data. We refer to Lemma 5.4 for the details.

1.4. Zero order estimates. For all results on the regularity of the spatial gradient from the preceding section, our methods yield analogues for the solution itself. These results hold under weaker conditions, namely we can allow an arbitrary Carathéodory function $a: \Omega_{T} \times \mathbf{R}^{n} \rightarrow \mathbf{R}^{n}$ with (1.6) and (1.7), in particular we neither need to impose continuity in the first nor differentiability with respect to the second argument. Furthermore, now we can include variational inequalities (1.12) containing a divergence term given by $F \in L^{2}\left(\Omega_{T}, \mathbf{R}^{n}\right)$, and we can drop the assumption $D^{2} \psi \in$ $L^{2}\left(\Omega_{T}\right)$. Under these conditions, there holds the zero order potential estimate

$$
\left|u\left(z_{0}\right)\right| \leq c\left(f_{C_{R}\left(z_{0}\right)}|u|^{2} d z\right)^{\frac{1}{2}}+c \mathbf{P}_{2}^{f, \psi}\left(z_{0}, R\right)+c \mathbf{P}_{1}^{F, \psi}\left(z_{0}, R\right)+c R
$$

for every Lebesgue point $z_{0} \in \Omega_{T}$ of $u$ and $0<R<\operatorname{dist}_{\mathcal{P}}\left(z_{0}, \partial \Omega_{T}\right)$, with the potentials

$$
\mathbf{P}_{2}^{f, \psi}\left(z_{0}, R\right):=\int_{0}^{R}\left[\frac{|f|^{2}\left(C_{\varrho}\left(z_{0}\right)\right)}{\varrho^{n-2}}\right]^{\frac{1}{2}} \frac{d \varrho}{\varrho}+\int_{0}^{R}\left[\frac{\left|\partial_{t} \psi\right|^{2}\left(C_{\varrho}\left(z_{0}\right)\right)}{\varrho^{n-2}}\right]^{\frac{1}{2}} \frac{d \varrho}{\varrho}
$$

and

$$
\mathbf{P}_{1}^{F, \psi}\left(z_{0}, R\right):=\int_{0}^{R}\left[\frac{|F|^{2}\left(C_{\varrho}\left(z_{0}\right)\right)}{\varrho^{n}}\right]^{\frac{1}{2}} \frac{d \varrho}{\varrho}+\int_{0}^{R}\left[\frac{|D \psi|^{2}\left(C_{\varrho}\left(z_{0}\right)\right)}{\varrho^{n}}\right]^{\frac{1}{2}} \frac{d \varrho}{\varrho} .
$$

For the proof, we refer to Theorem 4.1. As in the case of gradient estimates, the above estimate implies Lorentz space estimates for the solutions itself, see Corollary 4.2.

Moreover, we deduce a condition for continuity of the solution corresponding to (1.16). Namely, in dimensions $n>2$ there holds

$$
f, \partial_{t} \psi \in L\left(\frac{n+2}{2}, 1\right) \quad \text { and } \quad|F|,|D \psi| \in L(n+2,1) \quad \text { implies } \quad u \in C_{\mathrm{loc}}^{0}\left(\Omega_{T}\right),
$$


cf. Corollary 5.3. Finally, we mention that under a certain Morrey-type condition on the data, we are able to prove Hölder continuity of the solution, see Lemma 5.5.

The present article is organized as follows. After some preliminary remarks in the following section, we proceed to the excess decay estimate for solutions to obstacle problems in Section 3. This estimate is the key step both for the potential estimates derived in Section 4 and for the results on continuity established in Section 5. We conclude the article with an appendix, in which we present some results on the relationship between potentials and Lorentz spaces.

\section{Preliminaries}

Notation. The domain in space-time on which we will formulate our parabolic problems will be denoted by $\Omega_{T}:=\Omega \times(0, T)$, where $\Omega \subset \mathbf{R}^{n}$ is a bounded domain of dimension $n \geq 2$ and $T>0$. Subdomains will frequently be written as $\mathcal{O}_{I}:=\mathcal{O} \times I$, where $\mathcal{O} \subset \Omega$ and $I:=\left(t_{1}, t_{2}\right) \subset(0, T)$. The parabolic boundary of such a subset is defined by $\partial_{\mathcal{P}} \mathcal{O}_{I}:=\left(\mathcal{O} \times\left\{t_{1}\right\}\right) \cup\left(\partial \mathcal{O} \times\left[t_{1}, t_{2}\right]\right)$. For points in space-time, we use notations such as $z=(x, t)$ or $z_{0}=\left(x_{0}, t_{0}\right)$, where $x, x_{0} \in \Omega$ denote spatial variables and $t, t_{0} \in(0, T)$ the time variables. The standard parabolic cylinders will be abbreviated by

$$
C_{\varrho}\left(z_{0}\right):=B_{\varrho}\left(x_{0}\right) \times\left(t_{0}-\varrho^{2}, t_{0}+\varrho^{2}\right)
$$

for $z_{0}=\left(x_{0}, t_{0}\right) \in \mathbf{R}^{n} \times \mathbf{R}$ and $\varrho>0$, where here, $B_{\varrho}\left(x_{0}\right)$ denotes the open ball with radius $\varrho>0$ and center $x_{0}$. If the center is the origin, we will also use the more compact notations $C_{\varrho}:=C_{\varrho}(0)$ and $B_{\varrho}:=B_{\varrho}(0)$. The parabolic cylinders are the balls with respect to the parabolic metric $d_{\mathcal{P}}\left(z_{1}, z_{2}\right):=\max \left\{\left|x_{1}-x_{2}\right|, \sqrt{\left|t_{1}-t_{2}\right|}\right\}$ for $z_{i}=\left(x_{i}, t_{i}\right) \in \mathbf{R}^{n+1}, i=1,2$.

For the mean value of a function $f \in L^{1}\left(\Omega_{T}\right)$ over a subset $A \subset \Omega_{T}$, we abbreviate $f_{A}:=f_{A} f(z) d z$. In the case that $A=C_{\varrho}\left(z_{0}\right)$ is a parabolic cylinder, we will frequently write $f_{z_{0}, \varrho}=f_{C_{\varrho}\left(z_{0}\right)}$, or even shorter $f_{\varrho}=f_{C_{\varrho}}$ if the cylinder is centered in the origin.

Function spaces. We use the customary notation $W^{1,2}(\Omega)$ for the Sobolev space of weakly differentiable functions $f: \Omega \rightarrow \mathbf{R}$ with $|f|,|D f| \in L^{2}(\Omega)$ and write $W_{0}^{1,2}(\Omega)$ for the closure of $C_{\mathrm{cpt}}^{\infty}(\Omega)$ in $W^{1,2}(\Omega)$ with respect to the $W^{1,2}$-norm. With $W^{-1,2}(\Omega)$ we denote the dual space of $W_{0}^{1,2}(\Omega)$. The dual pairing between these spaces will be indicated by the brackets $\langle\cdot, \cdot\rangle$.

Next, we introduce parabolic function spaces. For a time $T>0$, an exponent $q \in[1, \infty]$ and a Banach space $B$, we employ the notation $L^{q}(0, T ; B)$ for the space of measurable functions $g:(0, T) \rightarrow B$ with

$$
\|g\|_{L^{q}(0, T ; B)}:=\left(\int_{0}^{T}\|g(t)\|_{B}^{q} d t\right)^{\frac{1}{q}}<\infty
$$

in case $q<\infty$, respectively with

$$
\|g\|_{L^{\infty}(0, T ; B)}:=\operatorname{ess~sup}_{t \in(0, T)}\|g(t)\|_{B}<\infty
$$

if $q=\infty$. For the space of continuous functions $g:[0, T] \rightarrow B$, equipped with the norm $\|\cdot\|_{L^{\infty}(0, T ; B)}$, we write $C^{0}([0, T] ; B)$.

The relevant spaces for parabolic problems with quadratic growth are in particular the spaces $C^{0}\left([0, T] ; L^{2}(\Omega)\right)$ and $L^{2}\left(0, T ; W^{1,2}(\Omega)\right)$. Moreover, the distributional 
space $L^{2}\left(0, T ; W^{-1,2}(\Omega)\right)$, which is the dual space to $L^{2}\left(0, T ; W_{0}^{1,2}(B)\right)$, plays a prominent role. In particular, the time derivatives of solutions $u$ to a parabolic equation with quadratic growth satisfy $\partial_{t} u \in L^{2}\left(0, T ; W^{-1,2}(\Omega)\right)$. However, the same regularity property fails to hold for general solutions to parabolic obstacle problems, which causes severe technical problems in their analysis.

Comparing sums and integrals. The following easy lemma will frequently be used throughout the proofs in order to estimate certain potentials by sums and vice versa.

Lemma 2.1. Assume that $\Phi:(0, R] \rightarrow[0, \infty)$ is a function that satisfies

$$
\Phi(\varrho) \leq K \Phi(r) \text { if } \theta r \leq \varrho \leq r \leq R,
$$

for constants $K \geq 1$ and $\theta \in(0,1)$. Then there holds

$$
\frac{|\log \theta|}{K} \sum_{\ell=1}^{\infty} \Phi\left(\theta^{\ell} R\right) \leq \int_{0}^{R} \Phi(\varrho) \frac{d \varrho}{\varrho} \leq K|\log \theta| \sum_{\ell=0}^{\infty} \Phi\left(\theta^{\ell} R\right) .
$$

Proof. For $r_{\ell}:=\theta^{\ell} R, \ell \in \mathbf{N}_{0}$, decompose the domain of integration into the intervals $\left(r_{\ell+1}, r_{\ell}\right)$ and use the estimate $\frac{1}{K} \Phi\left(r_{\ell+1}\right) \leq \Phi(\varrho) \leq K \Phi\left(r_{\ell}\right)$ for all $\varrho \in$ $\left(r_{\ell+1}, r_{\ell}\right)$ that holds by $(2.1)$.

\section{Excess decay estimates}

3.1. Estimates for comparison maps. We begin by stating excess decay estimates for parabolic equations, which we will use as comparison problems. We start with an estimate for a zero-order excess, which follows from classical theory (see $[6,24])$.

Lemma 3.1. Suppose that on a parabolic cylinder $C_{R}=B_{R} \times\left(-R^{2}, R^{2}\right)$, the function $w \in C^{0}\left(\left[-R^{2}, R^{2}\right] ; L^{2}\left(B_{R}\right)\right) \cap L^{2}\left(-R^{2}, R^{2} ; W^{1,2}\left(B_{R}\right)\right)$ weakly solves the parabolic equation

$$
-\operatorname{div} a(x, t, D w)=0 \quad \text { on } C_{R},
$$

under the assumptions (1.6) and (1.7). Then the solution satisfies $w \in C_{\mathrm{loc}}^{0, \beta}\left(C_{R}\right)$ for some exponent $\beta \in(0,1)$ depending at most on $n, \nu$ and $L$, and with a constant $c \geq 1$ depending on the same data, there holds the following excess decay estimate for all radii $\varrho \in(0, R)$

$$
f_{C_{\varrho}}\left|w-(w)_{\varrho}\right|^{2} d z \leq c\left(\frac{\varrho}{R}\right)^{2 \beta} f_{C_{R}}\left|w-(w)_{R}\right|^{2} d z+c R^{2} .
$$

Proof. It suffices to prove the assertion in the case $(w)_{R}=0$, since $w-(w)_{R}$ is again a solution to the same parabolic equation. From [24, Thm. 6.28], we infer the estimate

$$
\operatorname{Osc}_{C_{\varrho}} w \leq c\left(\frac{\varrho}{R}\right)^{\beta} \operatorname{osc}_{C_{R / 2}} w+c R \text { for all } \varrho \in\left(0, \frac{R}{2}\right),
$$

where $\operatorname{osc}_{C_{\varrho}} w:=\sup _{C_{\varrho}} w-\inf _{C_{\varrho}} w$ denotes the oscillation, and from [24, Thm. 6.17], we get

$$
\sup _{C_{R / 2}}|w|^{2} \leq c f_{C_{R}}|w|^{2} d z+c R^{2},
$$

with constants $\beta \in(0,1)$ and $c \geq 1$ depending only on $n, \nu$ and $L$. We point out that in order to derive the above estimates, only the growth assumptions (1.6) and 
(1.7) are needed and no linearity of the equation, cf. the remark at the beginning of Section VI.5 in [24]. Joining the above two estimates, we derive the bound

$f_{C_{\varrho}}\left|w-(w)_{\varrho}\right|^{2} d z \leq\left(\operatorname{osc}_{C_{\varrho}} w\right)^{2} \leq c\left(\frac{\varrho}{R}\right)^{2 \beta}\left(\operatorname{osc}_{C_{R / 2}} w\right)^{2}+c R^{2} \leq c\left(\frac{\varrho}{R}\right)^{2 \beta} f_{C_{R}}|w|^{2} d z+c R^{2}$

for all $\varrho \in\left(0, \frac{R}{2}\right)$. For $\varrho \in\left[\frac{R}{2}, R\right]$, the same estimate holds trivially. Since we have assumed $(w)_{R}=0$, this implies the claim.

Furthermore, we will need the corresponding decay estimate for the gradients of solutions.

Lemma 3.2. Assume that $w \in C^{0}\left(\left[-R^{2}, R^{2}\right] ; L^{2}\left(B_{R}\right)\right) \cap L^{2}\left(-R^{2}, R^{2} ; W^{1,2}\left(B_{R}\right)\right)$ is a weak solution to the parabolic equation

$$
\partial_{t} w-\operatorname{div} b(t, D w)=0,
$$

where the vector field $b:\left(-R^{2}, R^{2}\right) \times \mathbf{R}^{n} \rightarrow \mathbf{R}^{n}$ satisfies the assumptions (1.7), (1.8) and (1.9), and is independent from the spatial variable. Then, there holds the excess decay estimate

$$
f_{C_{\varrho}}\left|D w-(D w)_{\varrho}\right|^{2} d z \leq c\left(\frac{\varrho}{R}\right)^{2 \beta} f_{C_{R}}\left|D w-(D w)_{R}\right|^{2} d z
$$

for all radii $\varrho \in(0, R]$, where the constants $\beta \in(0,1)$ and $c \geq 1$ depend at most on $n, \nu$ and $L$.

Proof. A standard application of the difference quotient method yields the higher differentiability $\partial_{i} w \in L_{\text {loc }}^{2}\left(-R^{2}, R^{2} ; W_{\text {loc }}^{1,2}\left(B_{R}\right)\right)$ for all $i \in\{1, \ldots, n\}$, and furthermore, the partial derivatives weakly solve the parabolic equation

$$
\partial_{t}\left(\partial_{i} w\right)-\operatorname{div}\left(B(x, t) D \partial_{i} w\right)=0 \quad \text { on } C_{R / 2},
$$

where we defined $B(x, t):=D_{\xi} b(t, D w(x, t))$. By our assumptions (1.8) and (1.9), this defines a measurable function $B: C_{R} \rightarrow \mathbf{R}^{n \times n}$, which satisfies

$$
B(x, t) \zeta \cdot \zeta \geq \nu|\zeta|^{2} \text { and } \quad|B(x, t)| \leq L
$$

for all $(x, t) \in C_{R}$ and $\zeta \in \mathbf{R}^{n}$. Therefore, the same argument as in the proof of the preceding Lemma 3.1, see also [24, (6.42)], gives the estimate

$$
f_{C_{\varrho}}\left|\partial_{i} w-\left(\partial_{i} w\right)_{\varrho}\right|^{2} d z \leq c\left(\frac{\varrho}{R}\right)^{2 \beta} f_{C_{R}}\left|\partial_{i} w-\left(\partial_{i} w\right)_{R}\right|^{2} d z \leq c\left(\frac{\varrho}{R}\right)^{2 \beta} f_{C_{R}}\left|D w-(D w)_{R}\right|^{2} d z,
$$

for all radii $\varrho \in\left(0, \frac{R}{2}\right)$, and trivially also for radii $\varrho \in\left[\frac{R}{2}, R\right]$. Since here, the index $i \in\{1, \ldots, n\}$ is arbitrary, we derive the asserted estimate.

Next, we state two comparison estimates that we will employ to transfer the above estimates for homogeneous equations to the setting of an obstacle problem. The proofs can be found in [31] for problems with more general growth exponents $p>\frac{2 n}{n+2}$. We state the results here for the easier case $p=2$.

Lemma 3.3. [31, Lemma 3.5] Suppose that the assumptions (1.6) and (1.7) are in force, that the inhomogeneity satisfies $f \in L^{2}\left(t_{1}, t_{2} ; W^{-1,2}(\mathcal{O})\right)$ and that the obstacle function $\psi \in C^{0}\left(\left[t_{1}, t_{2}\right] ; L^{2}(\mathcal{O})\right) \cap L^{2}\left(t_{1}, t_{2} ; W^{1,2}(\mathcal{O})\right)$ satisfies $\partial_{t} \psi \in$ $L^{2}\left(t_{1}, t_{2} ; W^{-1,2}(\mathcal{O})\right)$. We assume that

$$
v \in C^{0}\left(\left[t_{1}, t_{2}\right] ; L^{2}(\mathcal{O})\right) \cap L^{2}\left(t_{1}, t_{2} ; W^{1,2}(\mathcal{O})\right) \quad \text { with } \quad \partial_{t} v \in L^{2}\left(t_{1}, t_{2} ; W^{-1,2}(\mathcal{O})\right)
$$


solves the parabolic equation

$$
\partial_{t} v-\operatorname{div} a(\cdot, D v)=\partial_{t} \psi-\operatorname{div} a(\cdot, D \psi) \quad \text { in } L^{2}\left(t_{1}, t_{2} ; W^{-1,2}(\mathcal{O})\right),
$$

and that $u \in K\left(\psi, v ; \mathcal{O}_{I}\right)$ is a solution of the variational inequality

$$
\begin{aligned}
& \int_{t_{1}}^{t_{2}}\left\langle\partial_{t} w, w-u\right\rangle d t+\int_{\mathcal{O}_{I}} a(z, D u) \cdot D(w-u) d z+\frac{1}{2}\left\|(w-u)\left(\cdot, t_{1}\right)\right\|_{L^{2}}^{2} \\
& \geq \int_{t_{1}}^{t_{2}}\langle f, w-u\rangle d t
\end{aligned}
$$

for all comparison functions $w \in K^{\prime}\left(\psi, v ; \mathcal{O}_{I}\right)$. Then there holds the comparison estimate

$$
\int_{\mathcal{O}_{I}}|D u-D v|^{2} d z \leq c(\nu)\|f\|_{L^{2}-W^{-1,2}\left(\mathcal{O}_{I}\right)}^{2}+c(\nu)\left\|\partial_{t} \psi-\operatorname{div} a(\cdot, D \psi)\right\|_{L^{2-W}-1,2\left(\mathcal{O}_{I}\right)}^{2} .
$$

Next, we state a standard comparison estimate for solutions of two parabolic equations.

Lemma 3.4. [31, Lemma 3.6] Assume that the vector fields $a, b: \Omega_{T} \times \mathbf{R}^{n} \rightarrow \mathbf{R}^{n}$ satisfy the growth assumption (1.7), and $b$ additionally the monotonicity property (1.6). Furthermore, let $g, h \in L^{2}\left(t_{1}, t_{2} ; W^{-1,2}(\mathcal{O})\right)$ and assume that

$$
v, w \in C^{0}\left(\left[t_{1}, t_{2}\right] ; L^{2}(\mathcal{O})\right) \cap L^{2}\left(t_{1}, t_{2} ; W^{1,2}(\mathcal{O})\right)
$$

are solutions of the parabolic equations

$$
\partial_{t} v-\operatorname{div} a(\cdot, D v)=g \quad \text { in } L^{2}\left(t_{1}, t_{2} ; W^{-1,2}(\mathcal{O})\right),
$$

respectively

$$
\partial_{t} w-\operatorname{div} b(\cdot, D w)=h \quad \text { in } L^{2}\left(t_{1}, t_{2} ; W^{-1,2}(\mathcal{O})\right)
$$

with $v=w$ on $\partial_{\mathcal{P}} \mathcal{O}_{I}$. Then, for a constant $c=c(\nu)$, there holds the comparison estimate

$$
\begin{aligned}
& \int_{\mathcal{O}_{I}}|D v-D w|^{2} d z \\
& \leq c \int_{\mathcal{O}_{I}}|a(z, D v)-b(z, D v)|^{2} d z+c \int_{t_{1}}^{t_{2}}\|g(\cdot, t)-h(\cdot, t)\|_{W^{-1,2}}^{2} d t .
\end{aligned}
$$

\subsection{Excess decay estimates for solutions to obstacle problems.}

Lemma 3.5. Assume that $u \in C^{0}\left([0, T] ; L^{2}(\Omega)\right) \cap L^{2}\left(0, T ; W^{1,2}(\Omega)\right)$ is a localizable solution to the obstacle problem (1.12) - in the sense of Definition 1.1-under the assumptions (1.6) and (1.7), and let $C_{R}\left(z_{0}\right) \subset \Omega_{T}$. Then, there holds the zero order excess decay estimate

$$
\begin{aligned}
f_{C_{\varrho}\left(z_{0}\right)} \mid u & -\left.(u)_{z_{0}, \varrho}\right|^{2} d z \leq c\left(\frac{\varrho}{R}\right)^{2 \beta} f_{C_{R}\left(z_{0}\right)}\left|u-(u)_{z_{0}, R}\right|^{2} d z+c R^{2} \\
& +c\left(\frac{R}{\varrho}\right)^{n+2} f_{C_{R}\left(z_{0}\right)} R^{2}|F|^{2}+R^{4}|f|^{2}+R^{2}|D \psi|^{2}+R^{4}\left|\partial_{t} \psi\right|^{2} d z
\end{aligned}
$$


for all $\varrho \in(0, R]$. If additionally, the vector field satisfies the conditions (1.8), (1.9) and the continuity property stated in (1.10) then we have the estimate

$$
\begin{aligned}
& f_{C_{\varrho}\left(z_{0}\right)}\left|D u-(D u)_{z_{0}, \varrho}\right|^{2} d z \\
& \leq c\left(\left(\frac{\varrho}{R}\right)^{2 \beta}+\omega^{2}(R)\left(\frac{R}{\varrho}\right)^{n+2}\right) f_{C_{R}\left(z_{0}\right)}\left|D u-(D u)_{z_{0}, R}\right|^{2} d z \\
& \quad+c\left(\frac{R}{\varrho}\right)^{n+2}\left\{\omega^{2}(R)\left(1+\left|(D u)_{z_{0}, R}\right|^{2}\right)+f_{C_{R}\left(z_{0}\right)}|F|^{2}+R^{2}|f|^{2} d z\right. \\
& \left.\quad+R^{2} f_{C_{R}\left(z_{0}\right)}\left|\partial_{t} \psi\right|^{2}+\left|D^{2} \psi\right|^{2} d z+\omega^{2}(R) f_{C_{R}\left(z_{0}\right)}|D \psi|^{2} d z\right\} .
\end{aligned}
$$

In both estimates, the constant $c \geq 1$ depends only on $n, \nu$ and $L$ and the exponent $\beta=\beta(n, \nu, L)$ is the same as in Lemma 3.1, respectively in Lemma 3.2.

Proof. We assume $z_{0}=0$ for notational convenience. The strategy of the proof is to carry over the decay estimates from Lemma 3.1, respectively Lemma 3.2 to the case of obstacle problems by a comparison argument consisting of the following steps.

Step 1: Comparison with the parabolic equation

$$
\begin{cases}\partial_{t} w_{1}-\operatorname{div} a\left(\cdot, D w_{1}\right)=\partial_{t} \psi-\operatorname{div} a(\cdot, D \psi) & \text { on } C_{R}, \\ w_{1}=u & \text { on } \partial_{\mathcal{P}} C_{R} .\end{cases}
$$

Step 2: Comparison with the homogeneous equation

$$
\begin{cases}\partial_{t} w_{2}-\operatorname{div} a\left(\cdot, D w_{2}\right)=0 & \text { on } C_{R} \\ w_{2}=u & \text { on } \partial_{\mathcal{P}} C_{R}\end{cases}
$$

Step 3 (only for gradient estimates): Comparison with the equation with frozen coefficients

$$
\begin{cases}\partial_{t} w_{3}-\operatorname{div} a\left(0, t, D w_{3}\right)=0 & \text { on } C_{R} \\ w_{3}=u & \text { on } \partial_{\mathcal{P}} C_{R}\end{cases}
$$

The existence of the solutions to the above comparison problems follows from classical results, see e.g. [27, 34]. We point out that for the existence of solutions with boundary values $u$, it is crucial that the localizable solution $u$ satisfies the extension property from Definition 1.1(i), cf. [31, Lemma 4.1]. Next, we will give the comparison estimates for each of the steps listed above.

The Comparison Lemma 3.3 yields the estimate

$$
\int_{C_{R}}\left|D u-D w_{1}\right|^{2} d z \leq c \int_{C_{R}}|F|^{2}+R^{2}|f|^{2} d z+c\left\|\partial_{t} \psi-\operatorname{div} a(\cdot, D \psi)\right\|_{L^{2-W^{-1,2}}}^{2}
$$

Applying the Comparison Lemma 3.4 with $g=\partial_{t} \psi-\operatorname{div} a(\cdot, D \psi), h=0$ and $b=a$, we get

$$
\int_{C_{R}}\left|D w_{1}-D w_{2}\right|^{2} d z \leq c\left\|\partial_{t} \psi-\operatorname{div} a(\cdot, D \psi)\right\|_{L^{2-} W^{-1,2}}^{2}
$$


Combining the two preceding estimates and applying Poincaré's inequality, we arrive at

$$
\begin{aligned}
R^{-2} \int_{C_{R}}\left|u-w_{2}\right|^{2} d z & \leq c \int_{C_{R}}\left|D u-D w_{2}\right|^{2} d z \\
& \leq c \int_{C_{R}}|F|^{2}+R^{2}|f|^{2} d z+c\left\|\partial_{t} \psi-\operatorname{div} a(\cdot, D \psi)\right\|_{L^{2-W}-1,2}^{2} \\
& \leq c \int_{C_{R}}|F|^{2}+R^{2}|f|^{2}+R^{2}\left|\partial_{t} \psi\right|^{2}+|D \psi|^{2} d z
\end{aligned}
$$

where for the last step we employed the embedding $L^{2}\left(B_{R}\right) \hookrightarrow W^{-1,2}\left(B_{R}\right)$ and the growth assumption (1.7). This is the required comparison estimate for the zero order decay estimate. For the gradient estimate, we need an additional step in order to "freeze the coefficients". For this purpose, we apply once again the Comparison Lemma 3.4, this time with the structure function $b(x, t, \xi)=a(0, t, \xi)$ and $g=h=0$. This leads us to

$$
\begin{aligned}
\int_{C_{R}}\left|D w_{2}-D w_{3}\right|^{2} d z & \leq c \int_{C_{R}}\left|a\left(x, t, D w_{2}\right)-a\left(0, t, D w_{2}\right)\right|^{2} d z \\
& \leq c L^{2} \omega^{2}(R) \int_{C_{R}} 1+\left|D w_{2}\right|^{2} d z
\end{aligned}
$$

where we applied the continuity assumption (1.10) in the last step. Estimating the right-hand side by (3.12) and keeping in mind that $\omega \leq 1$, we deduce

$$
\begin{aligned}
\int_{C_{R}}\left|D w_{2}-D w_{3}\right|^{2} d z \leq & c \omega^{2}(R) \int_{C_{R}} 1+|D u|^{2} d z+c \int_{C_{R}}|F|^{2}+R^{2}|f|^{2} d z \\
& +c\left\|\partial_{t} \psi-\operatorname{div} a(\cdot, D \psi)\right\|_{L^{2}-W^{-1,2} .}^{2}
\end{aligned}
$$

Combining this estimate with (3.12), we arrive at

$$
\begin{aligned}
\int_{C_{R}}\left|D u-D w_{3}\right|^{2} d z \leq & c \omega^{2}(R) \int_{C_{R}} 1+|D u|^{2} d z+c \int_{C_{R}}|F|^{2}+R^{2}|f|^{2} d z \\
& +c\left\|\partial_{t} \psi-\operatorname{div} a(\cdot, D \psi)\right\|_{L^{2-} W^{-1,2}}^{2} .
\end{aligned}
$$

Next, we use the growth assumption (1.9), the embedding $L^{2}\left(B_{R}\right) \hookrightarrow W^{-1,2}\left(B_{R}\right)$ and the continuity assumption (1.10) in order to estimate

$$
\begin{aligned}
& \|\operatorname{div} a(\cdot, D \psi)\|_{L^{2}-W^{-1,2}\left(C_{R}\right)} \\
& \leq\left\|\operatorname{div} a\left(x_{0}, \cdot, D \psi\right)\right\|_{L^{2-} W^{-1,2}\left(C_{R}\right)}+\left\|\operatorname{div}\left[a\left(x_{0}, \cdot, D \psi\right)-a(\cdot, \cdot, D \psi)\right]\right\|_{L^{2-W^{-1,2}}\left(C_{R}\right)} \\
& \leq c R\left\|D^{2} \psi\right\|_{L^{2}}+c \omega(R)\left(1+\|D \psi\|_{L^{2}}\right) .
\end{aligned}
$$

Plugging this into (3.14) and using the embedding $L^{2}\left(B_{R}\right) \hookrightarrow W^{-1,2}\left(B_{R}\right)$ for estimating the time derivative, we deduce

$$
\begin{aligned}
\int_{C_{R}}\left|D u-D w_{3}\right|^{2} d z \leq & c \omega^{2}(R) \int_{C_{R}} 1+|D u|^{2} d z+c \int_{C_{R}}|F|^{2}+R^{2}|f|^{2} d z \\
& +c R^{2} \int_{C_{R}}\left|\partial_{t} \psi\right|^{2}+\left|D^{2} \psi\right|^{2} d z+c \omega^{2}(R) \int_{C_{R}}|D \psi|^{2} d z .
\end{aligned}
$$


Proof of the zero order estimate. By Lemma 3.1, the solution $w_{2}$ of the homogeneous parabolic equation (3.10) satisfies an excess decay estimate of the form

$$
f_{C_{\varrho}}\left|w_{2}-\left(w_{2}\right)_{\varrho}\right|^{2} d z \leq c\left(\frac{\varrho}{R}\right)^{2 \beta} f_{C_{R}}\left|w_{2}-\left(w_{2}\right)_{R}\right|^{2} d z+c R^{2},
$$

for every $\varrho \in(0, R]$, where $\beta \in(0,1)$ and $c \geq 1$ are constants depending only on $n, \nu$ and $L$. In order to transfer this decay estimate to the solution $u$ of the obstacle problem, we calculate

$$
\begin{aligned}
f_{C_{\varrho}}\left|u-(u)_{\varrho}\right|^{2} d z & \leq f_{C_{\varrho}}\left|u-\left(w_{2}\right)_{\varrho}\right|^{2} d z \\
& \leq 2 f_{C_{\varrho}}\left|w_{2}-\left(w_{2}\right)_{\varrho}\right|^{2} d z+2 f_{C_{\varrho}}\left|u-w_{2}\right|^{2} d z \\
& \leq c\left(\frac{\varrho}{R}\right)^{2 \beta} f_{C_{R}}\left|w_{2}-\left(w_{2}\right)_{R}\right|^{2} d z+c R^{2}+2\left(\frac{R}{\varrho}\right)^{n+2} f_{C_{R}}\left|u-w_{2}\right|^{2} d z \\
& \leq c\left(\frac{\varrho}{R}\right)^{2 \beta} f_{C_{R}}\left|u-(u)_{R}\right|^{2} d z+c R^{2}+c\left(\frac{R}{\varrho}\right)^{n+2} f_{C_{R}}\left|u-w_{2}\right|^{2} d z .
\end{aligned}
$$

This implies the claimed zero order estimate by bounding the last integral with (3.13).

Proof of the gradient estimate. Here, we additionally impose the stronger assumptions (1.8), (1.9) and (1.10). In this situation, the solution $w_{3} \in L^{2}\left(-R^{2}, R^{2}\right.$; $\left.W^{1,2}\left(B_{R}\right)\right)$ of the parabolic equation $(3.11)$, whose coefficients are independent from the spatial variable, satisfies the following excess decay estimate, cf. Lemma 3.2.

$$
f_{C_{\varrho}}\left|D w_{3}-\left(D w_{3}\right)_{\varrho}\right|^{2} d z \leq c\left(\frac{\varrho}{R}\right)^{2 \beta} f_{C_{R}}\left|D w_{3}-\left(D w_{3}\right)_{R}\right|^{2} d z \quad \text { for all } \varrho \in(0, R],
$$

where $\beta \in(0,1)$ and $c \geq 1$ depend at most on $n, \nu$ and $L$. As above, this estimate implies the following decay estimate for the spatial gradient of the solution to the obstacle problem.

$$
\begin{aligned}
& f_{C_{\varrho}}\left|D u-(D u)_{\varrho}\right|^{2} d z \leq f_{C_{\varrho}}\left|D u-\left(D w_{3}\right)_{\varrho}\right|^{2} d z \\
& \quad \leq 2 f_{C_{\varrho}}\left|D w_{3}-\left(D w_{3}\right)_{\varrho}\right|^{2} d z+2 f_{C_{\varrho}}\left|D u-D w_{3}\right|^{2} d z \\
& \quad \leq c\left(\frac{\varrho}{R}\right)^{2 \beta} f_{C_{R}}\left|D w_{3}-\left(D w_{3}\right)_{R}\right|^{2} d z+2\left(\frac{R}{\varrho}\right)^{n+2} f_{C_{R}}\left|D u-D w_{3}\right|^{2} d z \\
& \quad \leq c\left(\frac{\varrho}{R}\right)^{2 \beta} f_{C_{R}}\left|D u-(D u)_{R}\right|^{2} d z+c\left(\frac{R}{\varrho}\right)^{n+2} f_{C_{R}}\left|D u-D w_{3}\right|^{2} d z
\end{aligned}
$$

From this we deduce the claim (3.9) by bounding the last integral by means of (3.15).

\section{Potential estimates and applications}

In this section, we will derive pointwise estimates for the solutions and their gradients by the following non-linear potentials. For the zero-order estimates, the 
influence of the inhomogeneities and the obstacle are given by the potentials

$$
\mathbf{P}_{2}^{f, \psi}\left(z_{0}, R\right):=\int_{0}^{R} \varrho^{2}\left[f_{C_{\varrho}\left(z_{0}\right)}|f|^{2}+\left|\partial_{t} \psi\right|^{2} d z\right]^{\frac{1}{2}} \frac{d \varrho}{\varrho}
$$

and

$$
\mathbf{P}_{1}^{F, \psi}\left(z_{0}, R\right):=\int_{0}^{R} \varrho\left[f_{C_{\varrho}\left(z_{0}\right)}|F|^{2}+|D \psi|^{2} d z\right]^{\frac{1}{2}} \frac{d \varrho}{\varrho} .
$$

For the gradient estimates, the relevant potential is

$$
\mathbf{P}_{1}^{f, \psi}\left(z_{0}, R\right):=\int_{0}^{R} \varrho\left[f_{C_{\varrho}\left(z_{0}\right)}|f|^{2}+\left|\partial_{t} \psi\right|^{2}+\left|D^{2} \psi\right|^{2} d z\right]^{\frac{1}{2}} \frac{d \varrho}{\varrho} .
$$

We recall that in this situation, we have to restrict ourselves to the case $F=0$. With the notation introduced above, our result reads as follows.

Theorem 4.1. Consider a localizable solution $u \in C^{0}\left([0, T] ; L^{2}(\Omega)\right) \cap L^{2}(0, T$; $\left.W^{1,2}(\Omega)\right)$ to the obstacle problem (1.12), in the sense of Definition 1.1, where the assumptions (1.6) and (1.7) are in force. In every Lebesgue point $z_{0} \in \Omega_{T}$ of $u$ and for every radius $R>0$ with $C_{R}\left(z_{0}\right) \subset \Omega_{T}$, there holds the estimate

$$
\left|u\left(z_{0}\right)\right| \leq c\left(f_{C_{R}\left(z_{0}\right)}|u|^{2} d z\right)^{\frac{1}{2}}+c \mathbf{P}_{2}^{f, \psi}\left(z_{0}, R\right)+c \mathbf{P}_{1}^{F, \psi}\left(z_{0}, R\right)+c R .
$$

For the gradient estimates, we additionally assume that the vector field a satisfies the stronger conditions (1.8), (1.9) and the Dini-continuity property as in (1.10) and (1.11). Moreover, we assume that $F \equiv 0$ and that the obstacle function satisfies $\psi \in W^{1,2}\left(0, T ; L^{2}(\Omega)\right) \cap L^{2}\left(0, T ; W^{2,2}(\Omega)\right)$. Then there is a radius $R_{0}>0$, depending only on $n, \nu, L$ and $\omega(\cdot)$, such that in every Lebesgue point $z_{0} \in \Omega_{T}$ of $D u$, there holds

$$
\left|D u\left(z_{0}\right)\right| \leq 1+c\left(f_{C_{R}\left(z_{0}\right)}|D u|^{2} d z\right)^{\frac{1}{2}}+c \mathbf{P}_{1}^{f, \psi}\left(z_{0}, R\right)+c \int_{0}^{R} \omega(\varrho)\left[f_{C_{\varrho}\left(z_{0}\right)}|D \psi|^{2} d z\right]^{\frac{1}{2}} \frac{d \varrho}{\varrho}
$$

for every radius $0<R<\min \left\{R_{0}\right.$, $\left.\operatorname{dist}_{\mathcal{P}}\left(z_{0}, \partial \Omega_{T}\right)\right\}$. In both estimates, the constant $c \geq 1$ depends at most on $n, \nu$ and $L$.

Proof of the zero order estimate. For a Lebesgue point $z_{0} \in \Omega_{T}$, we consider a radius $R \in\left(0, \operatorname{dist}_{\mathcal{P}}\left(z_{0}, \partial \Omega_{T}\right)\right)$ and fix a parameter $\theta \in(0,1)$ sufficiently small to ensure $c \theta^{2 \beta} \leq \frac{1}{8}$, where $\beta \in(0,1)$ and $c \geq 1$ are the constants from Lemma 3.5. With the notations $R_{\ell}:=\theta^{\ell} R$ and $C_{\ell}:=C_{R_{\ell}}\left(z_{0}\right)$ for $\ell \in \mathbf{N}$, we estimate for an arbitrary $m \in \mathbf{N}$

$$
\begin{aligned}
\left|(u)_{C_{m+1}}\right| & \leq\left|(u)_{C_{2}}\right|+\sum_{\ell=2}^{m}\left|(u)_{C_{\ell+1}}-(u)_{C_{\ell}}\right| \\
& \leq \frac{1}{\theta^{2(n+2)}}\left(f_{C_{R}\left(z_{0}\right)}|u|^{2} d z\right)^{\frac{1}{2}}+\frac{1}{\theta^{n+2}} \sum_{\ell=2}^{m}\left(f_{C_{\ell}}\left|u-(u)_{C_{\ell}}\right|^{2} d z\right)^{\frac{1}{2}} .
\end{aligned}
$$

For the estimate of the last sum, we apply the excess decay estimate from Lemma 3.5 with $\varrho=R_{\ell}$ and $R=R_{\ell-1}$, which yields for our choice of $\theta$

$$
f_{C_{\ell}}\left|u-(u)_{C_{\ell}}\right|^{2} d z \leq \frac{1}{8} f_{C_{\ell-1}}\left|u-(u)_{C_{\ell-1}}\right|^{2} d z+c R_{\ell-1}^{2}+c \theta^{-n-2} R_{\ell-1}^{2} \Phi\left(R_{\ell-1}\right),
$$


where we abbreviated

$$
\Phi(\varrho):=f_{C_{\varrho}\left(z_{0}\right)} \varrho^{2}|f|^{2}+\varrho^{2}\left|\partial_{t} \psi\right|^{2}+|F|^{2}+|D \psi|^{2} d z
$$

for all $\varrho \in(0, R)$. Taking square roots in (4.2) and summing over $\ell=2, \ldots, m$ we infer - since $\theta$ has been fixed in dependence on $n, \nu$ and $L$ - that there holds

$$
\begin{aligned}
& \sum_{\ell=2}^{m}\left(f_{C_{\ell}}\left|u-(u)_{C_{\ell}}\right|^{2} d z\right)^{\frac{1}{2}} \\
& \leq \frac{1}{2} \sum_{\ell=1}^{m-1}\left(f_{C_{\ell}}\left|u-(u)_{C_{\ell}}\right|^{2} d z\right)^{\frac{1}{2}}+c R \sum_{\ell=2}^{m} \theta^{\ell-1}+c \sum_{\ell=2}^{m}\left[R_{\ell-1}^{2} \Phi\left(R_{\ell-1}\right)\right]^{\frac{1}{2}} \\
& \leq \frac{1}{2} \sum_{\ell=2}^{m}\left(f_{C_{\ell}}\left|u-(u)_{C_{\ell}}\right|^{2} d z\right)^{\frac{1}{2}}+c\left(f_{C_{R}\left(z_{0}\right)}|u|^{2} d z\right)^{\frac{1}{2}}+c R+c \sum_{\ell=2}^{m}\left[R_{\ell-1}^{2} \Phi\left(R_{\ell-1}\right)\right]^{\frac{1}{2}} .
\end{aligned}
$$

Re-absorbing the first integral on the right-hand side and joining the resulting estimate with (4.1), we deduce that for all $m \in \mathbf{N}$, there holds

$$
\begin{aligned}
\left|(u)_{C_{m+1}}\right| & \leq c\left(f_{C_{R}\left(z_{0}\right)}|u|^{2} d z\right)^{\frac{1}{2}}+c R+c \sum_{\ell=2}^{m}\left[R_{\ell-1}^{2} \Phi\left(R_{\ell-1}\right)\right]^{\frac{1}{2}} \\
& \leq c\left(f_{C_{R}\left(z_{0}\right)}|u|^{2} d z\right)^{\frac{1}{2}}+c R+c \int_{0}^{R}\left[\varrho^{2} \Phi(\varrho)\right]^{\frac{1}{2}} \frac{d \varrho}{\varrho}
\end{aligned}
$$

where we applied Lemma 2.1 in the last step. Since $z_{0} \in \Omega_{T}$ was chosen as a Lebesgue point of $u$ and therefore $\left|u\left(z_{0}\right)\right|=\lim _{m \rightarrow \infty}\left|(u)_{C_{m+1}}\right|$, we conclude the claimed zero order estimate.

Proof of the gradient estimate. With the constants $c \geq 1$ and $\beta \in(0,1)$ from Lemma 3.5, we choose a parameter $\theta \in(0,1)$ in dependence on $n, \nu$ and $L$ so small that $c \theta^{2 \beta} \leq \frac{1}{16}$, and we will later choose the radius $R_{0} \in\left(0, \operatorname{dist}_{\mathcal{P}}\left(z_{0}, \partial \Omega_{T}\right)\right)$ small enough to ensure

$$
c \omega^{2}\left(R_{0}\right) \theta^{-n-2} \leq \frac{1}{16} .
$$

We fix a Lebesgue point $z_{0}=\left(x_{0}, t_{0}\right) \in \Omega_{T}$ of $D u$ and a radius $R \in\left(0, R_{0}\right)$. We continue to use the notations $R_{\ell}:=\theta^{\ell} R$ and $C_{\ell}:=C_{R_{\ell}}\left(z_{0}\right)$ for $\ell \in \mathbf{N}$. Analogously as in (4.1), we deduce

$$
\left|(D u)_{C_{m+1}}\right| \leq \frac{1}{\theta^{2(n+2)}}\left(f_{C_{R}\left(z_{0}\right)}|D u|^{2} d z\right)^{\frac{1}{2}}+\frac{1}{\theta^{n+2}} \sum_{\ell=2}^{m}\left(f_{C_{\ell}}\left|D u-(D u)_{C_{\ell}}\right|^{2} d z\right)^{\frac{1}{2}}
$$

for every $m \in \mathbf{N}$. The excess decay estimate (3.9) from Lemma 3.5 implies by our choices of $\theta$ and $R_{0}$ and since $F=0$ that

$$
\begin{aligned}
f_{C_{\ell}}\left|D u-(D u)_{C_{\ell}}\right|^{2} d z \leq & \frac{1}{8} f_{C_{\ell-1}}\left|D u-(D u)_{C_{\ell-1}}\right|^{2} d z \\
& +c \theta^{-n-2}\left[\omega^{2}\left(R_{\ell-1}\right)\left(1+\left|(D u)_{C_{\ell-1}}\right|^{2}\right)+\Phi\left(R_{\ell-1}\right)\right]
\end{aligned}
$$


holds for every $\ell \in \mathbf{N}$, where we re-defined the function $\Phi(\varrho)$ for $\varrho \in\left(0, R_{0}\right)$ by

$$
\Phi(\varrho):=\varrho^{2} f_{C_{\varrho}\left(z_{0}\right)}|f|^{2}+\left|\partial_{t} \psi\right|^{2}+\left|D^{2} \psi\right|^{2} d z+\omega^{2}(\varrho) f_{C_{\varrho}\left(z_{0}\right)}|D \psi|^{2} d z .
$$

Next, we take the square roots of the estimate (4.5) and sum over $\ell=2, \ldots, m$, with the result

$$
\begin{aligned}
\sum_{\ell=2}^{m}\left(f_{C_{\ell}}\left|D u-(D u)_{C_{\ell}}\right|^{2} d z\right)^{\frac{1}{2}} & \leq \frac{1}{2} \sum_{\ell=2}^{m}\left(f_{C_{\ell}}\left|D u-(D u)_{C_{\ell}}\right|^{2} d z\right)^{\frac{1}{2}} \\
+c\left(f_{C_{R}\left(z_{0}\right)}|D u|^{2} d z\right)^{\frac{1}{2}} & +c \sum_{\ell=1}^{m-1} \omega\left(R_{\ell}\right)\left(1+\left|(D u)_{C_{\ell}}\right|\right)+c \sum_{\ell=1}^{m-1}\left[\Phi\left(R_{\ell}\right)\right]^{\frac{1}{2}} .
\end{aligned}
$$

Here, we can re-absorb the first integral on the right-hand side. In view of (4.4), we thereby deduce

$$
\begin{aligned}
\left|(D u)_{C_{m+1}}\right| \leq & c\left(f_{C_{R}\left(z_{0}\right)}|D u|^{2} d z\right)^{\frac{1}{2}}+c \sum_{\ell=1}^{m-1} \omega\left(R_{\ell}\right)\left(1+\left|(D u)_{C_{\ell}}\right|\right)+c \sum_{\ell=1}^{m-1}\left[\Phi\left(R_{\ell}\right)\right]^{\frac{1}{2}} \\
\leq & c\left(f_{C_{R}\left(z_{0}\right)}|D u|^{2} d z\right)^{\frac{1}{2}}+c \int_{0}^{R}[\Phi(\varrho)]^{\frac{1}{2}} \frac{d \varrho}{\varrho} \\
& +c \max _{1 \leq \ell \leq m}\left(1+\left|(D u)_{C_{\ell}}\right|\right) \int_{0}^{R} \omega(\varrho) \frac{d \varrho}{\varrho}
\end{aligned}
$$

for all $m \in \mathbf{N}$, where the last step is a consequence of Lemma 2.1. At this stage, we use the Dini condition (1.11) on the modulus of continuity $\omega$ in order to fix the maximal radius $R_{0} \in\left(0, \operatorname{dist}\left(z_{0}, \partial \Omega_{T}\right)\right)$ so small that additionally to the condition (4.3), we have

$$
c \int_{0}^{R_{0}} \omega(\varrho) \frac{d \varrho}{\varrho} \leq \frac{1}{2},
$$

where $c$ denotes the constant from (4.6). With this choice of $R_{0}$, the estimate (4.6) implies

$$
\left|(D u)_{C_{m+1}}\right| \leq \frac{1}{2} \max _{1 \leq \ell \leq m}\left(1+\left|(D u)_{C_{\ell}}\right|\right)+c\left(f_{C_{R}\left(z_{0}\right)}|D u|^{2} d z\right)^{\frac{1}{2}}+c \int_{0}^{R}[\Phi(\varrho)]^{\frac{1}{2}} \frac{d \varrho}{\varrho},
$$

from which we infer inductively that

$$
\left|(D u)_{C_{m+1}}\right| \leq 1+2 c\left(f_{C_{R}\left(z_{0}\right)}|D u|^{2} d z\right)^{\frac{1}{2}}+2 c \int_{0}^{R}[\Phi(\varrho)]^{\frac{1}{2}} \frac{d \varrho}{\varrho}
$$

holds for every $m \in \mathbf{N}$. Since $z_{0}$ was assumed to be a Lebesgue point of $D u$, we arrive at

$$
\left|D u\left(z_{0}\right)\right|=\lim _{m \rightarrow \infty}\left|(D u)_{C_{m+1}}\right| \leq 1+c\left(f_{C_{R}\left(z_{0}\right)}|D u|^{2} d z\right)^{\frac{1}{2}}+c \int_{0}^{R}[\Phi(\varrho)]^{\frac{1}{2}} \frac{d \varrho}{\varrho} .
$$

By the definition of $\Phi$, this yields the claimed estimate (3.9).

The potential estimates from the preceding theorem, combined with classical estimates for potentials, imply estimates in various scales of function spaces. Here, we mention only the following results for the scale of Lorentz spaces, which refine the 
Calderón-Zygmund estimates from [31] in the case of Dini-continuous vector fields with growth exponent $p=2$.

Corollary 4.2. Suppose that $u \in C^{0}\left([0, T] ; L^{2}(\Omega)\right) \cap L^{2}\left(0, T ; W^{1,2}(\Omega)\right)$ is a localizable solution to the obstacle problem (1.12), under the assumptions (1.6) and (1.7), and let $N:=n+2$.

(i) If the data satisfies

$$
f, \partial_{t} \psi \in L^{2}(\Omega) \text { and }|F|,|D \psi| \in L\left(\frac{2 N}{N-2}, \infty\right)
$$

then in dimensions $n>2$, the solution satisfies $u \in L\left(\frac{2 N}{N-4}, \infty\right)\left(\mathcal{O}_{I}\right)$ for every subdomain $\mathcal{O}_{I} \Subset \Omega_{T}$ and we have the local estimate

$$
\begin{aligned}
& \|u\|_{L\left(\frac{2 N}{N-4}, \infty\right)\left(\mathcal{O}_{I}\right)} \\
& \leq c\left(1+\|u\|_{L^{2}}+\|f\|_{L^{2}}+\left\|\partial_{t} \psi\right\|_{L^{2}}+\|F\|_{L\left(\frac{2 N}{N-2}, \infty\right)}+\|D \psi\|_{L\left(\frac{2 N}{N-2}, \infty\right)}\right),
\end{aligned}
$$

with a constant $c=c\left(n, \nu, L,\left|\mathcal{O}_{I}\right|, \operatorname{dist}_{\mathcal{P}}\left(\mathcal{O}_{I}, \partial \Omega_{T}\right)\right)$. For $n=2$, this estimate remains valid if we replace the left-hand side by $\|u\|_{L^{q}\left(\mathcal{O}_{I}\right)}$ for an arbitrary exponent $q \in[1, \infty)$.

(ii) Assume that the data satisfies

$$
f, \partial_{t} \psi \in L(r, s) \text { and }|F|,|D \psi| \in L\left(\frac{N r}{N-r}, s\right)
$$

for $2<r<\frac{N}{2}$ and $1 \leq s \leq \infty$. Then the solution satisfies $u \in L\left(\frac{N r}{N-2 r}, s\right)\left(\mathcal{O}_{I}\right)$ for every subdomain $\mathcal{O}_{I} \Subset \Omega_{T}$ and there holds the local estimate

$$
\begin{aligned}
& \|u\|_{L\left(\frac{N r}{N-2 r}, s\right)\left(\mathcal{O}_{I}\right)} \\
& \leq c\left(1+\|u\|_{L^{2}}+\|f\|_{L(r, s)}+\left\|\partial_{t} \psi\right\|_{L(r, s)}+\|F\|_{L\left(\frac{N r}{N-r}, s\right)}+\|D \psi\|_{L\left(\frac{N r}{N-r}, s\right)}\right),
\end{aligned}
$$

where the constant $c$ depends only on $n, \nu, L,\left|\mathcal{O}_{I}\right|$ and $\operatorname{dist}_{\mathcal{P}}\left(\mathcal{O}_{I}, \partial \Omega_{T}\right)$.

Proof. For the proof of (i) in the case $n>2$, we apply Lemma 6.1 once with the parameter $\gamma=2$ in the borderline case $r=1$ to the functions $f$ and $\partial_{t} \psi$ and once more with $\gamma=1, r=\frac{N}{N-2}$ and $s=\infty$ to $|F|$ and $|D \psi|$. This yields

$$
\begin{aligned}
& \left\|\mathbf{P}_{2}^{f, \psi}(\cdot, R)\right\|_{L\left(\frac{2 N}{N-4}, \infty\right)} \leq c\left(\|f\|_{L^{2}}+\left\|\partial_{t} \psi\right\|_{L^{2}}\right) \\
& \left\|\mathbf{P}_{1}^{F, \psi}(\cdot, R)\right\|_{L\left(\frac{2 N}{N-4}, \infty\right)} \leq c\left(\|F\|_{L\left(\frac{2 N}{N-2}, \infty\right)}+\|D \psi\|_{L\left(\frac{2 N}{N-2}, \infty\right)}\right) .
\end{aligned}
$$

In view of Theorem 4.1, this implies the asserted estimate. In the case of spatial dimension $n=2$, we instead apply Lemma 6.1 for an arbitrary parameter $\gamma<2$ to $f$ and $\partial_{t} \psi$ in order to derive bounds for $\|u\|_{L^{q}}$ for an arbitrary $q \in[1, \infty)$.

For the proof of (ii), we proceed analogously, where here, we choose the parameters $\frac{r}{2}$ and $\frac{s}{2}$ in the Lorentz spaces in Lemma 6.1 in order to treat the functions $f$ and $\partial_{t} \psi$, while for the functions $F$ and $D \psi$, we choose $\frac{1}{2} \frac{N r}{N-r}$ and $\frac{s}{2}$. This gives

$$
\begin{aligned}
& \left\|\mathbf{P}_{2}^{f, \psi}(\cdot, R)\right\|_{L\left(\frac{N r}{N-2 r}, s\right)} \leq c\left(\|f\|_{L(r, s)}+\left\|\partial_{t} \psi\right\|_{L(r, s)}\right) \\
& \left\|\mathbf{P}_{1}^{F, \psi}(\cdot, R)\right\|_{L\left(\frac{N r}{N-2 r}, s\right)} \leq c\left(\|F\|_{L\left(\frac{N r}{N-r}, s\right)}+\|D \psi\|_{L\left(\frac{N r}{N-r}, s\right)}\right) .
\end{aligned}
$$

The potential estimates from Theorem 4.1 thereby yield the claimed estimates.

Next, we give the corresponding estimates on the level of the gradient. 
Corollary 4.3. Let $u \in C^{0}\left([0, T] ; L^{2}(\Omega)\right) \cap L^{2}\left(0, T ; W^{1,2}(\Omega)\right)$ be a localizable solution to the obstacle problem (1.12) with $F=0$, where (1.7), (1.8), (1.9), (1.10) and (1.11) are in force.

(i) Assume that there holds

$$
f, \partial_{t} \psi,\left|D^{2} \psi\right| \in L^{2}(\Omega) \text { and }|D \psi| \in L\left(\frac{2 N}{N-2}, \infty\right),
$$

where $N:=n+2$. Then we have $|D u| \in L\left(\frac{2 N}{N-2}, \infty\right)\left(\mathcal{O}_{I}\right)$ for every $\mathcal{O}_{I} \Subset$ $\Omega_{T}$. Moreover, for a constant $c=c\left(n, \nu, L, \omega(\cdot),\left|\mathcal{O}_{I}\right|\right.$, $\left.\operatorname{dist}_{\mathcal{P}}\left(\mathcal{O}_{I}, \partial \Omega_{T}\right)\right)$, there holds the local estimate

$$
\begin{aligned}
& \|D u\|_{L\left(\frac{2 N}{N-2}, \infty\right)\left(\mathcal{O}_{I}\right)} \\
& \leq c\left(1+\|D u\|_{L^{2}}+\|f\|_{L^{2}}+\left\|\partial_{t} \psi\right\|_{L^{2}}+\left\|D^{2} \psi\right\|_{L^{2}}+\|D \psi\|_{L\left(\frac{2 N}{N-2}, \infty\right)}\right) .
\end{aligned}
$$

(ii) Assume that there holds

$$
f, \partial_{t} \psi,\left|D^{2} \psi\right| \in L(r, s) \quad \text { and } \quad|D \psi| \in L\left(\frac{N r}{N-r}, s\right)
$$

for $2<r<N$ and $1 \leq s \leq \infty$. Then we have $|D u| \in L\left(\frac{N r}{N-r}, s\right)\left(\mathcal{O}_{I}\right)$ for every subdomain $\mathcal{O}_{I} \Subset \Omega_{T}$, and moreover, with a constant $c=c\left(n, \nu, L, \omega(\cdot),\left|\mathcal{O}_{I}\right|\right.$, $\left.\operatorname{dist}_{\mathcal{P}}\left(\mathcal{O}_{I}, \partial \Omega_{T}\right)\right)$,

$$
\begin{aligned}
& \|D u\|_{L\left(\frac{N r}{N-r}, s\right)\left(\mathcal{O}_{I}\right)} \\
& \leq c\left(1+\|D u\|_{L^{2}}+\|f\|_{L(r, s)}+\left\|\partial_{t} \psi\right\|_{L(r, s)}+\left\|D^{2} \psi\right\|_{L(r, s)}+\|D \psi\|_{L\left(\frac{N r}{N-r}, s\right)}\right) .
\end{aligned}
$$

Proof. We fix a subdomain $\mathcal{O}_{I} \Subset \Omega_{T}$ and the radius $R:=\frac{1}{2} \min \left\{R_{0}\right.$, $\operatorname{dist}_{\mathcal{P}}\left(\mathcal{O}_{I}\right.$, $\left.\left.\partial \Omega_{T}\right)\right\}$, where the radius $R_{0}$ is determined in Theorem 4.1 in dependence on $n, \nu, L$ and $\omega(\cdot)$. Theorem 4.1 provides us with the pointwise estimate

$$
\begin{aligned}
\left|D u\left(z_{0}\right)\right| \leq & 1+c R^{-1-n / 2}\|D u\|_{L^{2}}+c \int_{0}^{R} \omega(\varrho)\left[\frac{|D \psi|^{2}\left(C_{\varrho}\left(z_{0}\right)\right)}{\varrho^{N}}\right]^{\frac{1}{2}} \frac{d \varrho}{\varrho} \\
& +c \int_{0}^{R}\left[\frac{|f|^{2}\left(C_{\varrho}\left(z_{0}\right)\right)}{\varrho^{N-2}}\right]^{\frac{1}{2}}+\left[\frac{\left|\partial_{t} \psi\right|^{2}\left(C_{\varrho}\left(z_{0}\right)\right)}{\varrho^{N-2}}\right]^{\frac{1}{2}}+\left[\frac{\left|D^{2} \psi\right|^{2}\left(C_{\varrho}\left(z_{0}\right)\right)}{\varrho^{N-2}}\right]^{\frac{1}{2}} \frac{d \varrho}{\varrho}
\end{aligned}
$$

for any $z_{0} \in \mathcal{O}_{I}$, where $c=c(n, \nu, L)$. The potential in the last line can be bounded by applying Lemma 6.1 with $\gamma=1$ to each of the functions $f, \partial_{t} \psi$ and $D^{2} \psi$, analogously as in the preceding proof. It remains to estimate the first integral in (4.7). By Lemma 6.2 , it is bounded by

$$
\left\|\int_{0}^{R} \omega(\varrho)\left[\frac{|D \psi|^{2}\left(C_{\varrho}(\cdot)\right)}{\varrho^{N}}\right]^{\frac{1}{2}} \frac{d \varrho}{\varrho}\right\|_{L\left(\frac{N r}{N-r}, s\right)} \leq c\|D \psi\|_{L\left(\frac{N r}{N-r}, s\right)},
$$

for every $2<r<N$ and $1 \leq s \leq \infty$. This concludes the proof.

\section{Continuity results}

5.1. Sharp criteria for continuity. Here we give criteria which guarantee that the solution of the obstacle problem (1.12) is continuous, respectively of class $C^{1}$. We begin with the continuity results for the solutions. The proof consists of three steps. First, we establish local boundedness of $u$, then VMO-regularity and finally the desired continuity of the solution. The proof therefore gives sufficient 
conditions for boundedness, VMO-regularity and continuity that are listed in the theorem below.

Theorem 5.1. Let $u \in C^{0}\left([0, T] ; L^{2}(\Omega)\right) \cap L^{2}\left(0, T ; W^{1,2}(\Omega)\right)$ be a localizable solution of the obstacle problem (1.12) (cf. Definition 1.1), under the assumptions (1.6) and (1.7).

(i) Suppose that on every subdomain $\mathcal{O}_{I} \Subset \Omega_{T}$, we have the bound

$$
\sup _{z_{0} \in \mathcal{O}_{I}}\left[\mathbf{P}_{2}^{f, \psi}\left(z_{0}, R\right)+\mathbf{P}_{1}^{F, \psi}\left(z_{0}, R\right)\right]<\infty
$$

for some radius $0<R<\operatorname{dist}_{\mathcal{P}}\left(\mathcal{O}_{I}, \partial \Omega_{T}\right)$. Then, the solution satisfies $u \in$ $L_{\text {loc }}^{\infty}\left(\Omega_{T}\right)$.

(ii) If additionally to the assumptions in (i), there holds

$$
\lim _{\varrho \searrow 0} \sup _{z_{0} \in \mathcal{O}_{I}} f_{C_{\varrho}\left(z_{0}\right)} \varrho^{4}|f|^{2}+\varrho^{4}\left|\partial_{t} \psi\right|^{2}+\varrho^{2}|F|^{2}+\varrho^{2}|D \psi|^{2} d z=0
$$

for every subdomain $\mathcal{O}_{I} \Subset \Omega_{T}$, then there holds $u \in \operatorname{VMO}_{\text {loc }}\left(\Omega_{T}\right) \cap L_{\text {loc }}^{\infty}\left(\Omega_{T}\right)$.

(iii) If on every subdomain $\mathcal{O}_{I} \subset \Omega_{T}$, there holds

$$
\lim _{R \searrow 0} \sup _{z_{0} \in \mathcal{O}_{I}}\left[\mathbf{P}_{2}^{f, \psi}\left(z_{0}, R\right)+\mathbf{P}_{1}^{F, \psi}\left(z_{0}, R\right)\right]=0,
$$

then $u \in C_{\mathrm{loc}}^{0}\left(\Omega_{T}\right)$.

The corresponding statement for the gradients of the solutions reads as follows.

Theorem 5.2. We suppose that the monotonicity and growth assumptions (1.7), (1.8) and (1.9) are in force and that the Dini-continuity condition stated in (1.10) and (1.11) is valid. Under this set of assumptions, we consider a localizable solution $u \in C^{0}\left([0, T] ; L^{2}(\Omega)\right) \cap L^{2}\left(0, T ; W^{1,2}(\Omega)\right)$ of the obstacle problem (1.12).

(i) If on every subdomain $\mathcal{O}_{I} \Subset \Omega_{T}$, there holds

$$
\sup _{z_{0} \in \mathcal{O}_{I}}\left[\mathbf{P}_{1}^{f, \psi}\left(z_{0}, R\right)+\int_{0}^{R}\left(\omega^{2}(\varrho) f_{C_{\varrho}\left(z_{0}\right)}|D \psi|^{2} d z\right)^{\frac{1}{2}} \frac{d \varrho}{\varrho}\right]<\infty
$$

for some $0<R<\operatorname{dist}_{\mathcal{P}}\left(\mathcal{O}_{I}, \partial \Omega_{T}\right)$ then $D u \in L_{\text {loc }}^{\infty}\left(\Omega_{T}, \mathbf{R}^{n}\right)$.

(ii) Assume that additionally to the assumptions in (i), we have

$$
\lim _{\varrho \searrow 0} \sup _{z_{0} \in \mathcal{O}_{I}}\left[\varrho^{2} f_{C_{\varrho}\left(z_{0}\right)}|f|^{2}+\left|\partial_{t} \psi\right|^{2}+\left|D^{2} \psi\right|^{2} d z+\omega^{2}(\varrho) f_{C_{\varrho}\left(z_{0}\right)}|D \psi|^{2} d z\right]=0
$$

for every $\mathcal{O}_{I} \Subset \Omega_{T}$. Then $D u \in \operatorname{VMO}_{\text {loc }}\left(\Omega_{T}, \mathbf{R}^{n}\right) \cap L_{\text {loc }}^{\infty}\left(\Omega_{T}, \mathbf{R}^{n}\right)$.

(iii) If for every subdomain $\mathcal{O}_{I} \subset \Omega_{T}$, we have

$$
\lim _{R \searrow 0} \sup _{z_{0} \in \mathcal{O}_{I}}\left[\mathbf{P}_{1}^{f, \psi}\left(z_{0}, R\right)+\int_{0}^{R}\left(\omega^{2}(\varrho) f_{C_{\varrho}\left(z_{0}\right)}|D \psi|^{2} d z\right)^{\frac{1}{2}} \frac{d \varrho}{\varrho}\right]=0,
$$

then $u \in C_{\mathrm{loc}}^{1}\left(\Omega_{T}\right)$.

In view of Lemma 6.3, we readily deduce the following sufficient condition for the solutions to be continuous, respectively $C^{1}$, in terms of Lorentz spaces.

Corollary 5.3. Suppose that $u \in C^{0}\left([0, T] ; L^{2}(\Omega)\right) \cap L^{2}\left(0, T ; W^{1,2}(\Omega)\right)$ is a localizable solution to the obstacle problem (1.12), under the assumptions (1.6) and (1.7). Then we have: 
(i) If $n>2$ and the data satisfies

$$
f, \partial_{t} \psi \in L\left(\frac{n+2}{2}, 1\right), \quad \text { and }|F|,|D \psi| \in L(n+2,1),
$$

then the solution is continuous on $\Omega_{T}$.

(ii) Under the stronger growth assumption (1.9) and the Dini-continuity assumptions (1.10) and (1.11), the conditions $F=0$,

$$
f, \partial_{t} \psi,\left|D^{2} \psi\right| \in L(n+2,1) \quad \text { and } \quad|D \psi| \in L_{\mathrm{loc}}^{\infty}\left(\Omega_{T}\right)
$$

imply $u \in C_{\mathrm{loc}}^{1}\left(\Omega_{T}\right)$. Here, in the case of a vector-field $a(x, t, \xi)=a(t, \xi)$ without $x$-dependence, the assumption $|D \psi| \in L_{\text {loc }}^{\infty}\left(\Omega_{T}\right)$ can be omitted.

Since the proofs of the above two theorems are almost identical, we only give the proof of the gradient estimates in detail and then shortly sketch the necessary modifications for the proof of the zero order results.

Proof of Theorem 5.2. We fix a subdomain $\mathcal{O}_{I}=\mathcal{O} \times\left(t_{1}, t_{2}\right) \Subset \Omega_{T}$ and define another subdomain $\widetilde{\mathcal{O}_{I}}=\widetilde{\mathcal{O}} \times\left(t_{0}, t_{3}\right)$ with $\mathcal{O}_{I} \Subset \widetilde{\mathcal{O}_{I}} \Subset \Omega_{T}$ by letting

$$
\widetilde{\mathcal{O}}:=\left\{x \in \Omega: \operatorname{dist}(x, \partial \Omega)>\frac{1}{2} \operatorname{dist}(\mathcal{O}, \partial \Omega)\right\},
$$

$t_{0}:=\frac{1}{2} t_{1}$ and $t_{3}:=\frac{1}{2}\left(t_{2}+T\right)$. We begin with the

Proof of (i): Local boundedness. Joining the potential estimate from Theorem 4.1 with assumption (5.3) for the domain $\widetilde{\mathcal{O}_{I}}$ instead of $\mathcal{O}_{I}$, we infer

$$
M_{u}:=\sup _{z \in \widetilde{\mathcal{O}_{I}}}|D u(z)|<\infty .
$$

Since the domain $\mathcal{O}_{I} \subset \widetilde{\mathcal{O}_{I}}$ was arbitrary, we deduce the first claim $D u \in L_{\text {loc }}^{\infty}\left(\Omega_{T}, \mathbf{R}^{n}\right)$.

Proof of (ii): VMO-regularity. For every $0<R<\operatorname{dist}_{\mathcal{P}}\left(\mathcal{O}_{I}, \Omega_{T} \backslash \widetilde{\mathcal{O}_{I}}\right)$, we define

$$
\delta(R):=\sup _{0<\varrho \leq R} \sup _{z_{0} \in \mathcal{O}_{I}}\left[\varrho^{2} f_{C_{\varrho}\left(z_{0}\right)}|f|^{2}+\left|\partial_{t} \psi\right|^{2}+\left|D^{2} \psi\right|^{2} d z+\omega^{2}(\varrho) f_{C_{\varrho}\left(z_{0}\right)}|D \psi|^{2} d z\right],
$$

which satisfies $\delta(R) \rightarrow 0$ as $R \searrow 0$ by assumption (5.4). Keeping in mind the supremum bound (5.6), we deduce from Lemma 3.5 that

$$
\begin{aligned}
& f_{C_{\varrho}\left(z_{0}\right)}\left|D u-(D u)_{z_{0}, \varrho}\right|^{2} d z \\
& \quad \leq c\left(\left(\frac{\varrho}{R}\right)^{2 \beta}+\omega^{2}(R)\left(\frac{R}{\varrho}\right)^{n+2}\right) M_{u}^{2}+c\left(\frac{R}{\varrho}\right)^{n+2}\left(\omega^{2}(R)\left(1+M_{u}^{2}\right)+\delta(R)\right)
\end{aligned}
$$

for every $z_{0} \in \mathcal{O}_{I}$ and $\varrho \in(0, R]$, where the constants $\beta \in(0,1)$ and $c \geq 1$ depend at most on $n, \nu$ and $L$. For an arbitrary $\varepsilon>0$, we choose first a parameter $\theta \in(0,1)$ and then a radius $0<R_{0}<\operatorname{dist}_{\mathcal{P}}\left(\mathcal{O}_{I}, \Omega_{T} \backslash \widetilde{\mathcal{O}_{I}}\right)$ sufficiently small to make sure that

$$
c \theta^{2 \beta} M_{u}^{2} \leq \frac{\varepsilon^{2}}{2} \quad \text { and } \quad c \omega^{2}\left(R_{0}\right) \frac{M_{u}^{2}}{\theta^{n+2}}+\frac{c}{\theta^{n+2}}\left(\omega^{2}\left(R_{0}\right)\left(1+M_{u}^{2}\right)+\delta\left(R_{0}\right)\right) \leq \frac{\varepsilon^{2}}{2} .
$$

Letting $\varrho=\theta R$ in (5.7) we thus deduce

$$
f_{C_{\theta R}\left(z_{0}\right)}\left|D u-(D u)_{z_{0}, \theta R}\right| d z \leq\left(f_{C_{\theta R}\left(z_{0}\right)}\left|D u-(D u)_{z_{0}, \theta R}\right|^{2} d z\right)^{\frac{1}{2}} \leq \varepsilon
$$

for every $z_{0} \in \mathcal{O}_{I}$ and every $R \in\left(0, R_{0}\right]$. Since $\varepsilon>0$ can be chosen arbitrarily, this implies $D u \in \operatorname{VMO}\left(\mathcal{O}_{I}\right)$ and thus the claim (ii). 
Proof of (iii): Gradient continuity. The idea of the proof is to show that the convergence of the mean values $(D u)_{w, \varrho} \rightarrow D u(w)$ as $\varrho \searrow 0$ is uniform in $w \in \mathcal{O}_{I}$, which implies the continuity of the gradient. Before we proceed to the proof, we introduce the notation

$$
\Phi(w, \varrho):=\varrho^{2} f_{C_{\varrho}(w)}|f|^{2}+\left|\partial_{t} \psi\right|^{2}+\left|D^{2} \psi\right|^{2} d z+\omega^{2}(\varrho) f_{C_{\varrho}(w)}|D \psi|^{2} d z
$$

for every $w \in \mathcal{O}_{I}$ and $0<\varrho<\operatorname{dist}_{\mathcal{P}}\left(\mathcal{O}_{I}, \Omega_{T} \backslash \widetilde{\mathcal{O}_{I}}\right)$. Now we first fix a constant $\theta \in(0,1)$ and then a radius $0<R_{0}<\operatorname{dist}_{\mathcal{P}}\left(\mathcal{O}_{I}, \Omega_{T} \backslash \widetilde{\mathcal{O}_{I}}\right)$ so small that $c \theta^{2 \beta} \leq \frac{1}{16}$ and $c \omega^{2}\left(R_{0}\right) \theta^{-n-2} \leq \frac{1}{16}$, where $\beta \in(0,1)$ and $c \geq 1$ denote the constants from Lemma 3.5. Using the short-hand notation $R_{\ell}:=\theta^{\ell} R$ and $C_{\ell}:=C_{\ell}(w):=C_{R_{\ell}}(w)$ for $\ell \in \mathbf{N}$ and $w \in \mathcal{O}_{I}$, we can estimate

$$
\begin{aligned}
\left|(D u)_{C_{m}}-(D u)_{C_{k}}\right| & \leq \sum_{\ell=k}^{m-1}\left|(D u)_{C_{\ell+1}}-(D u)_{C_{\ell}}\right| \\
& \leq c(\theta) \sum_{\ell=k}^{m-1}\left(f_{C_{\ell}}\left|D u-(D u)_{C_{\ell}}\right|^{2} d z\right)^{\frac{1}{2}}
\end{aligned}
$$

for all $k, m \in \mathbf{N}$ with $k<m$, where we omitted the point $w \in \mathcal{O}_{I}$ for the sake of notational convenience. Next, we use the excess decay estimate from Lemma 3.5 in order to estimate the right-hand side further. In view of our choices of $\theta$ and $R_{0}$, this yields

$$
\begin{aligned}
& \sum_{\ell=k}^{m-1}\left(f_{C_{\ell}}\left|D u-(D u)_{C_{\ell}}\right|^{2} d z\right)^{\frac{1}{2}} \\
& \leq \frac{1}{2} \sum_{\ell=k-1}^{m-2}\left(f_{C_{\ell}}\left|D u-(D u)_{C_{\ell}}\right|^{2} d z\right)^{\frac{1}{2}}+c \sum_{\ell=k-1}^{m-2} \omega\left(R_{\ell}\right)\left(1+\left|(D u)_{C_{\ell}}\right|\right)+c \sum_{\ell=k-1}^{m-2}\left[\Phi\left(w, R_{\ell}\right)\right]^{\frac{1}{2}} \\
& \leq \frac{1}{2} \sum_{\ell=k}^{m-1}\left(f_{C_{\ell}}\left|D u-(D u)_{C_{\ell}}\right|^{2} d z\right)^{\frac{1}{2}}+\frac{1}{2}\left(f_{C_{k-1}}\left|D u-(D u)_{C_{k-1}}\right|^{2} d z\right)^{\frac{1}{2}} \\
& \quad+c\left(1+M_{u}\right) \int_{0}^{R_{k-2}} \omega(\varrho) \frac{d \varrho}{\varrho}+c \int_{0}^{R_{k-2}}[\Phi(w, \varrho)]^{\frac{1}{2}} \frac{d \varrho}{\varrho},
\end{aligned}
$$

where we applied (5.6) and Lemma 2.1 for the last estimate. Next, we re-absorb the first integral from the right-hand side into the left-hand side and conclude

$$
\begin{aligned}
& \sum_{\ell=k}^{m-1}\left(f_{C_{\ell}(w)}\left|D u-(D u)_{C_{\ell}(w)}\right|^{2} d z\right)^{\frac{1}{2}} \\
& \leq\left(f_{C_{k-1}(w)}\left|D u-(D u)_{C_{k-1}(w)}\right|^{2} d z\right)^{\frac{1}{2}}+c\left(1+M_{u}\right) \int_{0}^{R_{k-2}} \omega(\varrho) \frac{d \varrho}{\varrho} \\
& \quad+c \mathbf{P}_{1}^{f, \psi}\left(w, R_{k-2}\right)+c \int_{0}^{R_{k-2}}\left(\omega^{2}(\varrho) f_{C_{\varrho}\left(z_{0}\right)}|D \psi|^{2} d z\right)^{\frac{1}{2}} \frac{d \varrho}{\varrho}
\end{aligned}
$$

by definition of $\mathbf{P}_{1}^{f, \psi}$. From the VMO-regularity established in Step 2, the Dini continuity condition (1.11) and the assumption (5.5), we infer that the right-hand 
side of the preceding estimate vanishes in the limit $k \rightarrow \infty$, uniformly in $w \in \mathcal{O}_{I}$. Keeping in mind the estimate (5.8), we deduce

$$
\lim _{k, m \rightarrow \infty} \sup _{w \in \mathcal{O}_{I}}\left|(D u)_{C_{m}(w)}-(D u)_{C_{k}(w)}\right|=0,
$$

which means that $\left\{(D u)_{C_{m}(w)}\right\}_{m \in \mathbf{N}}$ is a uniform Cauchy sequence. By Lebesgue's differentiation theorem, the gradient $D u(w)=\lim _{m \rightarrow \infty}(D u)_{C_{m}(w)}$ therefore is the uniform limit of the continuous functions $w \mapsto(D u)_{C_{m}(w)}$, which yields the asserted continuity of $D u$.

Sketch of the proof of Theorem 5.1. For a given subdomain $\mathcal{O}_{I} \Subset \Omega_{T}$, we choose a subset $\widetilde{\mathcal{O}_{I}}$ with $\mathcal{O}_{I} \Subset \widetilde{\mathcal{O}_{I}} \Subset \Omega_{T}$. The proof of the local boundedness of $u$ is a straightforward consequence of the potential estimates from Theorem 4.1. In order to derive the VMO-regularity, we define $M_{0}:=\sup _{\mathcal{O}_{I}}|u|<\infty$ and

$$
\delta(R):=\sup _{0<\varrho \leq R} \sup _{z_{0} \in \mathcal{O}_{I}} f_{C_{\varrho}\left(z_{0}\right)} \varrho^{4}|f|^{2}+\varrho^{2}|F|^{2}+\varrho^{4}\left|\partial_{t} \psi\right|^{2}+\varrho^{2}|D \psi|^{2} d z
$$

for all $0<R<\operatorname{dist}_{\mathcal{P}}\left(\mathcal{O}_{I}, \Omega_{T} \backslash \widetilde{\mathcal{O}_{I}}\right)$, which satisfies $\delta(R) \rightarrow 0$ as $R \searrow 0$ by assumption (5.1). Then we apply Lemma 3.5, with the result

$$
f_{C_{\theta R}\left(z_{0}\right)}\left|u-(u)_{z_{0}, \theta R}\right|^{2} d z \leq c \theta^{2 \beta} M_{0}+c R^{2}+c \theta^{-n-2} \delta(R),
$$

where here, the right-hand side becomes arbitrarily small for all $z_{0} \in \mathcal{O}_{I}$ and $R \in$ $\left(0, R_{0}\right)$ if we choose first $\theta \in(0,1)$ and then $0<R_{0}<\operatorname{dist}_{\mathcal{P}}\left(\mathcal{O}_{I}, \Omega_{T} \backslash \widetilde{\mathcal{O}_{I}}\right)$ small enough. This implies the local VMO-regularity of $u$.

For the proof of the continuity, we choose a parameter $\theta \in(0,1)$ with $c \theta^{2 \beta} \leq \frac{1}{8}$, where $\beta$ and $c$ are as in Lemma 3.5. Then we proceed analogously as in the derivation of (5.8) and (5.9), but this time using the zero order decay estimate (3.8) from Lemma 3.5. We thereby deduce

$$
\begin{aligned}
& \left|(u)_{C_{m}(w)}-(u)_{C_{k}(w)}\right| \leq c \sum_{\ell=k}^{m-1}\left(f_{C_{\ell}(w)}\left|u-(u)_{C_{\ell}(w)}\right|^{2} d z\right)^{\frac{1}{2}} \\
& \quad \leq\left(f_{C_{k-1}(w)}\left|u-(u)_{C_{k-1}(w)}\right|^{2} d z\right)^{\frac{1}{2}}+c R_{k-1}+c \mathbf{P}_{2}^{f, \psi}\left(w, R_{k-2}\right)+c \mathbf{P}_{1}^{F, \psi}\left(w, R_{k-2}\right),
\end{aligned}
$$

for all $k<m$ in $\mathbf{N}$ and $w \in \mathcal{O}_{I}$. Here, the first integral on the right-hand side vanishes in the limit $k \rightarrow \infty$ uniformly in $w \in \mathcal{O}_{I}$, because of the VMO-regularity established above, and the potentials $\mathbf{P}_{2}^{f, \psi}$ and $\mathbf{P}_{1}^{F, \psi}$ converge to zero uniformly in $w \in \mathcal{O}_{I}$ by our assumption (5.2). This implies uniform convergence $(u)_{C_{m}(w)} \rightarrow u(w)$ on $\mathcal{O}_{I}$ and thereby the continuity of $u$.

\subsection{Hölder continuity and $\mathrm{C}^{1, \alpha}$-regularity.}

Lemma 5.4. Suppose that $u \in C^{0}\left([0, T] ; L^{2}(\Omega)\right) \cap L^{2}\left(0, T ; W^{1,2}(\Omega)\right)$ is a localizable solution to the obstacle problem (1.12) with $F=0$, where the structure function satisfies (1.7), (1.8), (1.9) and the continuity condition (1.10) for a Hölder-continuity modulus $\omega(\varrho):=\min \left\{1, \varrho^{\gamma}\right\}$ with $\gamma \in(0,1)$. Moreover, we assume $|D \psi| \in L_{\text {loc }}^{\infty}\left(\Omega_{T}\right)$ and that the Morrey-type condition

$$
\sup _{z_{0} \in \mathcal{O}_{I}} \sup _{0<\varrho<1} \varrho^{2-2 \gamma} f_{C_{\varrho}\left(z_{0}\right) \cap \Omega_{T}}|f|^{2}+\left|\partial_{t} \psi\right|^{2}+\left|D^{2} \psi\right|^{2} d z<\infty
$$


holds for every subset $\mathcal{O}_{I} \Subset \Omega$. Then the solution has a Hölder-continuous gradient $D u \in C_{\text {loc }}^{0, \alpha}\left(\Omega_{T}, \mathbf{R}^{n}\right)$ for every $0<\alpha<\min \{\beta, \gamma\}$, where $\beta=\beta(n, \nu, L) \in(0,1)$ denotes the exponent from Lemma 3.2.

Proof. For a fixed subset $\mathcal{O}_{I} \Subset \Omega_{T}$ we choose a subset $\mathcal{O}_{I}^{*}$ with $\mathcal{O}_{I} \Subset \mathcal{O}_{I}^{*} \Subset \Omega_{T}$ and let

$$
K^{2}:=\sup _{z_{0} \in \mathcal{O}_{I}^{*}} \sup _{0<\varrho<\varrho_{0}} \varrho^{2-2 \gamma} f_{C_{\varrho}\left(z_{0}\right)}|f|^{2}+\left|\partial_{t} \psi\right|^{2}+\left|D^{2} \psi\right|^{2} d z+f_{C_{\varrho}\left(z_{0}\right)}|D \psi|^{2} d z<\infty
$$

with $\varrho_{0}:=\frac{1}{2} \operatorname{dist}_{\mathcal{P}}\left(\mathcal{O}_{I}, \partial \Omega_{T}\right)$. By the potential estimate from Theorem 4.1, this implies

$$
\|D u\|_{L^{\infty}\left(\mathcal{O}_{I}^{*}\right)} \leq c\left(\|D u\|_{L^{2}}+K+1\right),
$$

where here and in the remainder of the proof, we write $c$ for universal constants that depend at most on $\alpha, \gamma, n, \nu, L,\left|\Omega_{T}\right|$ and $\operatorname{dist}_{\mathcal{P}}\left(\mathcal{O}_{I}, \partial \Omega_{T}\right)$. With the constant $c=c(n, \nu, L) \geq 1$ from Lemma 3.5 and a Hölder exponent $\alpha$ with $0<\alpha<\min \{\beta, \gamma\}$, we choose first a parameter $\theta=\theta(\alpha, \gamma, n, \nu, L)$ so small that

$$
c \theta^{2 \beta} \leq \frac{1}{2} \theta^{2 \alpha}
$$

and then a radius $0<R_{0} \leq \min \left\{1, \varrho_{0}, \frac{1}{2} \operatorname{dist}_{\mathcal{P}}\left(\mathcal{O}_{I}, \Omega_{T} \backslash \mathcal{O}_{I}^{*}\right)\right\}$ in dependence on the data $\alpha, \gamma, n, \nu, L$ and $\operatorname{dist}_{\mathcal{P}}\left(\mathcal{O}_{I}, \partial \Omega_{T}\right)$ with the property

$$
c \omega^{2}\left(R_{0}\right) \theta^{-n-2} \leq \frac{1}{2} \theta^{2 \alpha} \text {. }
$$

With these choices of $\theta$ and $R_{0}$ and the excess functional

$$
E\left(z_{0}, \varrho\right):=f_{C_{\varrho}\left(z_{0}\right)}\left|D u-(D u)_{z_{0}, \varrho}\right|^{2} d z
$$

for all $z_{0} \in \mathcal{O}_{I}$ and $\varrho \in\left(0, R_{0}\right)$, Lemma 3.5 implies the excess decay estimate

$$
\begin{aligned}
E\left(z_{0}, \theta R\right) \leq & \theta^{2 \alpha} E\left(z_{0}, R\right)+c \theta^{-n-2}\left\{\omega^{2}(R)\left(1+\|D u\|_{L^{\infty}\left(\mathcal{O}_{I}^{*}\right)}^{2}\right)\right. \\
& \left.\quad+R^{2} f_{C_{R}\left(z_{0}\right)}|f|^{2}+\left|\partial_{t} \psi\right|^{2}+\left|D^{2} \psi\right|^{2} d z+\omega^{2}(R) f_{C_{R}\left(z_{0}\right)}|D \psi|^{2} d z\right\} \\
\leq & \theta^{2 \alpha} E\left(z_{0}, R\right)+c R^{2 \gamma}\left(\|D u\|_{L^{2}}^{2}+K^{2}+1\right)
\end{aligned}
$$

for all $z_{0} \in \mathcal{O}_{I}$ and $R \in\left(0, R_{0}\right)$. Here, the last estimate is a consequence of $\omega(R)=$ $R^{\gamma}$, the bound (5.10) and the definition of $K$. Iterating the above estimate, we get

$$
E\left(z_{0}, \theta^{k} R\right) \leq \theta^{2 \alpha k}\left[E\left(z_{0}, R\right)+c R^{2 \gamma} \sum_{\ell=0}^{\infty} \theta^{2 \ell(\gamma-\alpha)}\left(\|D u\|_{L^{2}}^{2}+K^{2}+1\right)\right]
$$

for all $z_{0} \in \mathcal{O}_{I}$ and $k \in \mathbf{N}$. Here, the series on the right-hand side is finite since $\alpha<\gamma$ and $\theta \in(0,1)$. By a standard argument, we thus deduce

$$
f_{C_{r}\left(z_{0}\right)}\left|D u-(D u)_{z_{0}, r}\right|^{2} d z \leq c\left(\frac{r}{R}\right)^{2 \alpha}\left[\|D u\|_{L^{2}}^{2}+R^{2 \gamma}\left(K^{2}+1\right)\right]
$$

for all $0<r<R<R_{0}$ and $z_{0} \in \mathcal{O}_{I}$. Now the characterization of Hölder continuous functions by Campanato-Da Prato [8] yields $D u \in C^{0, \alpha}\left(\mathcal{O}_{I}, \mathbf{R}^{n}\right)$, as desired.

The analogous proof, now based on the zero order estimate from Lemma 3.5, also yields the following criterion for the Hölder continuity of the solution itself. We state it without proof in order to avoid a repetition of arguments. 
Lemma 5.5. Consider a localizable solution $u \in C^{0}\left([0, T] ; L^{2}(\Omega)\right) \cap L^{2}(0, T$; $W^{1,2}(\Omega)$ ) to the obstacle problem (1.12), where the assumptions (1.6) and (1.7) are in force and the data satisfies the Morrey estimate

$$
\sup _{z_{0} \in \mathcal{O}_{I}} \sup _{0<\varrho<1}\left[\varrho^{2-2 \gamma} f_{C_{\varrho}\left(z_{0}\right) \cap \Omega_{T}}|F|^{2}+|D \psi|^{2} d z+\varrho^{4-2 \gamma} f_{C_{\varrho}\left(z_{0}\right) \cap \Omega_{T}}|f|^{2}+\left|\partial_{t} \psi\right|^{2} d z\right]<\infty
$$

for some $\gamma \in(0,1)$ and every subset $\mathcal{O}_{I} \Subset \Omega$. Then there holds $u \in C_{\text {loc }}^{0, \alpha}\left(\Omega_{T}\right)$ for every $0<\alpha<\min \{\beta, \gamma\}$, where $\beta=\beta(n, \nu, L) \in(0,1)$ denotes the exponent from Lemma 3.1.

\section{Appendix: Parabolic potentials and Lorentz spaces}

Throughout this section, we consider measurable functions $f: \mathbf{R}^{n+1} \rightarrow \mathbf{R}$. However, everything applies as well to functions $f: \Omega_{T} \rightarrow \mathbf{R}$ defined on an arbitrary domain $\Omega_{T} \subset \mathbf{R}^{n+1}$ by extending the function by zero outside of $\Omega_{T}$.

Lorentz spaces. The non-increasing rearrangement of a function $f: \mathbf{R}^{n+1} \rightarrow \mathbf{R}$ is defined by

$$
f^{*}:[0, \infty) \rightarrow[0, \infty), \quad f^{*}(s):=\sup \{t \geq 0:|\{|f|>t\}|>s\} .
$$

For $1 \leq p<\infty$ and $0<q<\infty$, the Lorentz space $L(p, q)$ is defined as the space of measurable functions $f: \mathbf{R}^{n+1} \rightarrow \mathbf{R}$ for which the expressions

$$
\|f\|_{L(p, q)}:=\left(\int_{0}^{\infty}\left[r^{1 / p} f^{*}(r)\right]^{q} \frac{d r}{r}\right)^{\frac{1}{q}}
$$

are finite. These expressions define norms on $L(p, q)$ for all parameters $q \geq 1$. Moreover, the space $L(p, \infty)$, also known as Marcinkiewicz space, is defined analogously via the norm

$$
\|f\|_{L(p, \infty)}:=\sup _{r>0} r^{1 / p} f^{*}(r)
$$

For local variants of Lorentz spaces on a set $A \subset \mathbf{R}^{n}$ we write $L(p, q)(A)$ for the space of functions $f: A \rightarrow \mathbf{R}$ with $f \mathbf{1}_{A} \in L(p, q)$. Another way to define the Lorentz spaces is to consider the averaged versions of $f^{*}$, i.e. $f^{* *}(r):=f_{0}^{r} f^{*}(s) d s$ for all $r \geq 0$, and to use

$$
[f]_{p, q}:=\left(\int_{0}^{\infty}\left[r^{1 / p} f^{* *}(r)\right]^{q} \frac{d r}{r}\right)^{\frac{1}{q}}, \quad \text { respectively }[f]_{p, \infty}:=\sup _{r>0} r^{1 / p} f^{* *}(r),
$$

instead of (6.1), respectively (6.2) for the definition of $L(p, q)$. This leads to an equivalent definition of Lorentz spaces for the range of parameters $1<p<\infty$ and $1 \leq q \leq \infty$ in the sense that

$$
\|f\|_{L(p, q)} \leq[f]_{p, q} \leq c(p, q)\|f\|_{L(p, q)} .
$$

Here, the first inequality is an immediate consequence of the monotonicity of $f^{*}$ and the second one follows from Hardy's inequality, see e.g. [35, Lemma V.3.1]. For a more detailed exposition of the properties of Lorentz spaces we refer to Ziemer's book [37]. 
Parabolic potentials. We introduce the localized parabolic Riesz potential

$$
\mathbf{I}_{\alpha}^{f}\left(z_{0}, R\right):=\int_{0}^{R} \frac{|f|\left(C_{\varrho}\left(z_{0}\right)\right)}{\varrho^{n+2-\alpha}} \frac{d \varrho}{\varrho}
$$

for $\alpha \in(0, n+2]$, where $|f|\left(C_{\varrho}\left(z_{0}\right)\right):=\int_{C_{\varrho}\left(z_{0}\right)}|f| d z$. This potential can be estimated by the classical parabolic Riesz potential

$$
I_{\alpha}^{\mathcal{P}}(f)\left(z_{0}\right):=\int_{\mathbf{R}^{n+1}} \frac{|f(z)|}{d_{\mathcal{P}}\left(z, z_{0}\right)^{n+2-\gamma}} d z
$$

where $d_{\mathcal{P}}\left((x, t),\left(x_{0}, t_{0}\right)\right):=\max \left\{\left|x-x_{0}\right|, \sqrt{\left|t-t_{0}\right|}\right\}$. More precisely, an application of Fubini's theorem implies the bound

$$
\mathbf{I}_{\alpha}^{f}\left(x_{0}, R\right) \leq \frac{1}{n+2-\alpha} I_{\alpha}^{\mathcal{P}}(f)\left(x_{0}\right)
$$

independently from $R>0$, provided $\alpha \in(0, n+2)$. Next, we consider potentials adapted to parabolic problems with quadratic growth. For a parameter $\gamma \in\left(0, \frac{n+2}{2}\right]$ and a function $f \in L_{\text {loc }}^{2}\left(\mathbf{R}^{n+1}\right)$, we define a parabolic potential by

$$
\mathbf{P}_{\gamma}^{f}\left(z_{0}, R\right):=\int_{0}^{R}\left[\frac{|f|^{2}\left(C_{\varrho}\left(z_{0}\right)\right)}{\varrho^{n+2-2 \gamma}}\right]^{\frac{1}{2}} \frac{d \varrho}{\varrho}
$$

for $z_{0} \in \mathbf{R}^{n+1}$ and $R>0$, where we abbreviated $|f|^{2}\left(C_{\varrho}\left(z_{0}\right)\right):=\int_{C_{\varrho}\left(z_{0}\right)}|f|^{2} d x$. We claim that these potentials can be bounded by iterated Riesz potentials of HavinMaz'ya-type in the sense

$$
\mathbf{P}_{\gamma}^{f}\left(z_{0}, R\right) \leq c(n, \gamma) I_{\alpha}^{\mathcal{P}}\left(\left[I_{\alpha}^{\mathcal{P}}\left(|f|^{2}\right)\right]^{\frac{1}{2}}\right)\left(z_{0}\right) \quad \text { with } \alpha:=\frac{2}{3} \gamma
$$

for all $z_{0} \in \mathbf{R}^{n}, R>0$, and $\gamma \in\left(0, \frac{n+2}{2}\right]$. Iterated Riesz potentials as above were introduced in the fundamental works $[2,15]$. The preceding estimate can be checked in the following way:

$$
\begin{aligned}
\mathbf{P}_{\gamma}^{f}\left(z_{0}, R\right) & =2^{(n+2-\alpha) / 2} \int_{0}^{R} \varrho^{\alpha}\left[\frac{|f|^{2}\left(C_{\varrho}\left(z_{0}\right)\right)}{(2 \varrho)^{n+2-\alpha}}\right]^{\frac{1}{2}} \frac{d \varrho}{\varrho} \\
& \leq 2^{(n+2-\alpha) / 2} \int_{0}^{R} \varrho^{\alpha} f_{C_{\varrho}\left(z_{0}\right)}\left[\int_{C_{2 \varrho}(z)} \frac{|f(w)|^{2}}{d_{\mathcal{P}}(z, w)^{n+2-\alpha}} d w\right]^{\frac{1}{2}} d z \frac{d \varrho}{\varrho} \\
& \leq \frac{2^{(n+2-\alpha) / 2}}{\left|B^{n}\right|} \int_{0}^{R} \varrho^{\alpha-n-2} \int_{C_{\varrho}\left(z_{0}\right)}\left[I_{\alpha}^{\mathcal{P}}\left(|f|^{2}\right)(z)\right]^{\frac{1}{2}} d z \frac{d \varrho}{\varrho}
\end{aligned}
$$

Estimating the right-hand side by (6.4), we arrive at the claimed estimate (6.6).

6.1. Integrability estimates. In the Lorentz spaces defined above, there holds the following estimate for Riesz potentials.

$$
\left\|I_{\alpha}^{\mathcal{P}}(f)\right\|_{L\left(\frac{N r}{N-\alpha r}, s\right)} \leq c(n)\|f\|_{L(r, s)}
$$

for every $1<r<\infty, 1 \leq s \leq \infty$ and $0<\alpha<\frac{N}{r}$, where $N:=n+2$. Moreover, we have the borderline result

$$
\left\|I_{\alpha}^{\mathcal{P}}(f)\right\|_{L\left(\frac{N}{N-\alpha}, \infty\right)} \leq c(n)\|f\|_{L^{1}}
$$


for every $0<\alpha<N$. Both estimates follow from a convolution inequality in Lorentz spaces [37, Thm. 2.10.2]. In view of (6.6), we infer the following estimate for potentials of the type (6.5).

Lemma 6.1. Assume that for $1<r<\infty$ and $\frac{1}{2} \leq s \leq \infty$, we have $f \in$ $L(2 r, 2 s)$. Furthermore, let $0<\gamma<\frac{N}{2 r}$ be given, where $N:=n+2$. Then there holds $\mathbf{P}_{\gamma}^{f}(\cdot, R) \in L\left(2 \frac{N r}{N-2 \gamma r}, 2 s\right)$ and we have the corresponding estimate

$$
\left\|\mathbf{P}_{\gamma}^{f}(\cdot, R)\right\|_{L\left(2 \frac{N r}{N-2 \gamma r}, 2 s\right)} \leq c(n, \gamma)\|f\|_{L(2 r, 2 s)}
$$

for every $R>0$. If $f \in L^{2}\left(\mathbf{R}^{n}\right)$ and $0<\gamma<\frac{N}{2}$, we still have the estimate

$$
\left\|\mathbf{P}_{\gamma}^{f}(\cdot, R)\right\|_{L\left(2 \frac{N}{N-2 \gamma}, \infty\right)} \leq c(n, \gamma)\|f\|_{L^{2}} .
$$

Proof. In a first step, we restrict ourselves to parameters $1 \leq s \leq \infty$. The Riesz potential estimate (6.7) with $\alpha:=\frac{2}{3} \gamma$ implies

$$
\left\|\left[I_{\alpha}^{\mathcal{P}}\left(|f|^{2}\right)\right]^{\frac{1}{2}}\right\|_{L\left(2 \frac{N r}{N-\alpha r}, 2 s\right)} \leq c\left\||f|^{2}\right\|_{L(r, s)}^{\frac{1}{2}} \leq c\|f\|_{L(2 r, 2 s)} .
$$

Applying (6.6) and then once more the Riesz potential estimate (6.7) leads us to

$$
\left\|\mathbf{P}_{\gamma}^{f}(\cdot, R)\right\|_{L\left(2 \frac{N r}{N-2 \gamma r}, 2 s\right)} \leq c\left\|\left[I_{\alpha}^{\mathcal{P}}\left(|f|^{2}\right)\right]^{\frac{1}{2}}\right\|_{L\left(2 \frac{N r}{N-\alpha r}, 2 s\right)} \leq c\|f\|_{L(2 r, 2 s)}
$$

for every $R>0$, by the definition of $\alpha$. This yields the claim (6.9) provided $s \geq 1$. The case $s \in\left[\frac{1}{2}, 1\right)$ then follows by an application of the Marcinkiewicz interpolation theorem (see e.g. [3, Thm. IV.4.13]), using the quasi-linearity of the operator $f \mapsto$ $\mathbf{P}_{\gamma}^{f}(\cdot, R)$.

The second claim (6.10) follows analogously, now replacing (6.11) by the estimate

$$
\left\|\left[I_{\alpha}^{\mathcal{P}}\left(|f|^{2}\right)\right]^{\frac{1}{2}}\right\|_{L\left(2 \frac{N}{N-\alpha}, \infty\right)} \leq c\left\||f|^{2}\right\|_{L^{1}}^{\frac{1}{2}}=c\|f\|_{L^{2}}
$$

which follows from (6.8). Combining this with (6.7), we arrive at the claim (6.10).

Moreover, we state a result for the borderline case $\alpha=0$ of the preceding potential estimates.

Lemma 6.2. Suppose that $R>0$ and $\omega:[0, \infty) \rightarrow[0, \infty)$ is measurable with $\int_{0}^{R} \omega(\varrho) \frac{d \varrho}{\varrho}<\infty$. For $f \in L_{\text {loc }}^{2}\left(\mathbf{R}^{n+1}\right)$ and $z_{0} \in \mathbf{R}^{n+1}$, we let

$$
T f\left(z_{0}\right):=\int_{0}^{R} \omega(\varrho)\left[\frac{|f|^{2}\left(C_{\varrho}\left(z_{0}\right)\right)}{\varrho^{n+2}}\right]^{\frac{1}{2}} \frac{d \varrho}{\varrho} .
$$

This defines a bounded quasi-linear operator $T: L^{2}\left(\mathbf{R}^{n+1}\right) \rightarrow L^{2}\left(\mathbf{R}^{n+1}\right)$ and $T: L(r, s)$ $\rightarrow L(r, s)$ for every $2<r \leq \infty$ and $1 \leq s \leq \infty$, with the corresponding estimates

$$
\|T f\|_{L^{2}} \leq c\|f\|_{L^{2}} \text { and }\|T f\|_{L(r, s)} \leq c\|f\|_{L(r, s)} .
$$

Here, the constants depend on $n, R, \omega(\cdot)$ and in the second estimate additionally on $r$ and $s$.

Proof. We abbreviate $M:=\int_{0}^{R} \omega(\varrho) \frac{d \varrho}{\varrho}$. For any $q \geq 2$, we use Jensen's inequality, once with the measure $\frac{\omega(\varrho)}{M} \frac{d \varrho}{\varrho}$ and in the case $q>2$ once more with the measure 
$\left|B_{\varrho}\right|^{-1} d x$, which gives

$$
\begin{aligned}
|T f(w)|^{q} & \leq M^{q-1}\left|B_{1}\right|^{q / 2} \int_{0}^{R} \omega(\varrho)\left(f_{C_{\varrho}(w)}|f(z)|^{2} d z\right)^{\frac{q}{2}} \frac{d \varrho}{\varrho} \\
& \leq M^{q-1}\left|B_{1}\right|^{q / 2} \int_{0}^{R} \omega(\varrho) f_{C_{\varrho}(w)}|f(z)|^{q} d z \frac{d \varrho}{\varrho} .
\end{aligned}
$$

Integrating over $w \in \mathbf{R}^{n+1}$ and applying Fubini's theorem, we deduce

$$
\int_{\mathbf{R}^{n+1}}|T f(w)|^{q} d w \leq M^{q-1}\left|B_{1}\right|^{q / 2} \int_{0}^{R} \omega(\varrho) \frac{d \varrho}{\varrho} \int_{\mathbf{R}^{n+1}}|f(z)|^{q} d z=M^{q}\left|B_{1}\right|^{q / 2}\|f\|_{L^{q}}^{q} .
$$

We have thereby shown that $T$ defines a bounded operator $T: L^{q}\left(\mathbf{R}^{n+1}\right) \rightarrow L^{q}\left(\mathbf{R}^{n+1}\right)$ for every $q \geq 2$. This implies in particular the first asserted estimate, and by an application of the Marcinkiewicz interpolation theorem [3, Thm. IV.4.13], the operator $T$ is also bounded on the Lorentz spaces $L(r, s)$ as long as $r>2$.

6.2. Continuity estimates. Here, we provide a condition in terms of Lorentz spaces that ensures that the potentials uniformly tend to zero in the limit $R \searrow 0$. This yields sufficient conditions for the continuity of solutions to obstacle problems.

Lemma 6.3. For every $\gamma \in\left(0, \frac{N}{2}\right)$ there holds the estimate

$$
\sup _{z \in \mathbf{R}^{n+1}} \mathbf{P}_{\gamma}^{f}(z, R) \leq c(n, \gamma) \int_{0}^{\left|C_{R}\right|} \varrho^{\frac{\gamma}{N}} f^{*}(\varrho) \frac{d \varrho}{\varrho}
$$

for every $R>0$. In particular, for functions $f \in L\left(\frac{N}{\gamma}, 1\right)$ there holds

$$
\lim _{R \searrow 0} \sup _{z \in \mathbf{R}^{n+1}} \mathbf{P}_{\gamma}^{f}(z, R)=0 .
$$

Proof. By the definition of the non-increasing rearrangement, we have

$$
|f|^{2}\left(C_{\varrho}\left(z_{0}\right)\right)=\int_{C_{\varrho}\left(z_{0}\right)}|f|^{2} d y \leq \int_{0}^{\left|C_{\varrho}\right|}\left(|f|^{2}\right)^{*} d s=\omega_{n} \varrho^{N}\left(|f|^{2}\right)^{* *}\left(\omega_{n} \varrho^{N}\right)
$$

for all $z_{0} \in \mathbf{R}^{n+1}$ and $\varrho>0$, where we write $\omega_{n}:=\left|B^{n}\right|$. Consequently, we have

$$
\begin{aligned}
\int_{0}^{R}\left[\frac{|f|^{2}\left(C_{\varrho}\left(z_{0}\right)\right)}{\varrho^{N-2 \gamma}}\right]^{\frac{1}{2}} \frac{d \varrho}{\varrho} & \leq c(n) \int_{0}^{R}\left[\varrho^{2 \gamma}\left(|f|^{2}\right)^{* *}\left(\omega_{n} \varrho^{N}\right)\right]^{\frac{1}{2}} \frac{d \varrho}{\varrho} \\
& =c(n, \gamma) \int_{0}^{\left|C_{R}\right|}\left[\sigma^{\frac{2 \gamma}{N}}\left(|f|^{2}\right)^{* *}(\sigma)\right]^{\frac{1}{2}} \frac{d \sigma}{\sigma}
\end{aligned}
$$

by the transformation $\sigma=\omega_{n} \varrho^{N}$. Here, we wish to replace the term $\left(|f|^{2}\right)^{* *}$ in the above estimate by $\left(|f|^{2}\right)^{*}=\left(f^{*}\right)^{2}$. Using the monotonicity of $f^{*}$, we can use an elementary estimate for integrals of non-increasing functions (cf. e.g. [33, Lemma 2.4]) with the result

$$
\begin{aligned}
\int_{0}^{\left|C_{R}\right|}\left[\sigma^{\frac{2 \gamma}{N}}\left(|f|^{2}\right)^{* *}(\sigma)\right]^{\frac{1}{2}} \frac{d \sigma}{\sigma} & =\int_{0}^{\left|C_{R}\right|} \sigma^{\frac{1}{2}\left(\frac{2 \gamma}{N}-1\right)}\left[\int_{0}^{\sigma}\left(f^{*}\right)^{2}(s) d s\right]^{\frac{1}{2}} \frac{d \sigma}{\sigma} \\
& \leq 2 \int_{0}^{\left|C_{R}\right|} \sigma^{\frac{\gamma}{N}-\frac{1}{2}} \int_{0}^{\sigma} s^{\frac{1}{2}} f^{*}(s) \frac{d s}{s} \frac{d \sigma}{\sigma}
\end{aligned}
$$


Joining the last two estimates, using Fubini's theorem and the assumption $\gamma<N$, we infer

$$
\begin{aligned}
\int_{0}^{R}\left[\frac{|f|^{2}\left(C_{\varrho}\left(z_{0}\right)\right)}{\varrho^{N-2 \gamma}}\right]^{\frac{1}{2}} \frac{d \varrho}{\varrho} & \leq c(n, \gamma) \int_{0}^{\left|C_{R}\right|} \int_{s}^{\left|C_{R}\right|} \sigma^{\frac{\gamma}{N}-\frac{1}{2}} \frac{d \sigma}{\sigma} \cdot s^{\frac{1}{2}} f^{*}(s) \frac{d s}{s} \\
& \leq c(n, \gamma) \int_{0}^{\left|C_{R}\right|} s^{\frac{\gamma}{N}} f^{*}(s) \frac{d s}{s}
\end{aligned}
$$

which yields the asserted estimate. Finally, we suppose that $f \in L\left(\frac{N}{\gamma}, 1\right)$, or equivalently

$$
\int_{0}^{\infty} \varrho^{\frac{\gamma}{N}} f^{*}(\varrho) \frac{d \varrho}{\varrho}<\infty
$$

This implies that the right-hand side of (6.12) vanishes in the limit $R \searrow 0$, which proves the second claim.

\section{References}

[1] Acerbi, E., and G. Mingione: Gradient estimates for a class of parabolic systems. - Duke Math. J. 136:2, 2007, 285-320.

[2] Adams, D., and N. Meyers: Thinness and Wiener criteria for non-linear potentials. - Indiana Univ. Math. J. 22:2, 1972, 169-197.

[3] Bennett, C., and R. Sharpley: Interpolation of operators. - Pure Appl. Math. 129, Academic Press, Boston, MA, 1988.

[4] Bensoussan, A., and J. Lions: Applications of variational inequalities in stochastic control. - Stud. Math. Appl. 12, North-Holland Publ., Amsterdam-New York, 1982.

[5] Bögelein, V., F. DuzaAr, and G. Mingione: Degenerate problems with irregular obstacles. - J. Reine Angew. Math. 650, 2011, 107-160.

[6] Campanato, S.: Equazioni paraboliche del secondo ordine e spazi $\mathcal{L}^{2, \theta}(\Omega, \delta)$. - Ann. Mat. Pura Appl. (4) 73, 1966, 55-102.

[7] Cianchi, A.: Maximizing the $L^{\infty}$ norm of the gradient of solutions to the Poisson equation. - J. Geom. Anal. 2:6, 1992, 499-515.

[8] Da Prato, G.: Spazi $\mathcal{L}^{p, \vartheta}(\Omega, \delta)$ e loro proprietà. - Ann. Mat. Pura Appl. (4) 69, 1965, 383-392.

[9] DiBenedetto, E.: Degenerate parabolic equations. - Universitext, Springer, New York, 1993.

[10] Duzanr, F., and G. Mingione: Gradient estimates via non-linear potentials. - Amer. J. Math. 133:3, 2011, 1093-1149.

[11] DuzaAr, F., and G. Mingione: Gradient continuity estimates. - Calc. Var. Partial Differential Equations 39, 2010, 379-418.

[12] Duzane, F., and G. Mingione: Local Lipschitz regularity for degenerate elliptic systems. Ann. Inst. H. Poincaré Anal. Non Linéaire 27, 2010, 1361-1396.

[13] Duzane, F., and G. Mingione: Gradient estimates via linear and nonlinear potentials. - J. Funct. Anal. 259, 2010, 2961-2998.

[14] Duzane, F., G. Mingione, and K. Steffen: Parabolic systems with polynomial growth and regularity. - Mem. Amer. Math. Soc. 214:1005, 2011.

[15] Havin, V., and V. Maz'Ya: A nonlinear potential theory. - Russian Math. Surveys 27:6, 1972, $67-138$.

[16] Heinonen, J., T. Kilpeläinen, and O. Martio: Nonlinear potential theory of degenerate elliptic equations. - Oxford Math. Monogr., Oxford Univ. Press, New York, 1993. 
[17] Jin, T., V. Maz'Ya, and J. Van Schaftingen: Pathological solutions to elliptic problems in divergence form with continuous coefficients. - C. R. Math. Acad. Sci. Paris 347:13-14, 2009, $773-778$.

[18] Kilpeläinen, T., and P. Lindqvist: On the Dirichlet boundary value problem for a degenerate parabolic equation. - SIAM J. Math. Anal. 27:3, 1996, 661-683.

[19] Kilpeläinen, T., and J. MalÝ: Degenerate elliptic equations with measure data and nonlinear potentials. - Ann. Sc. Norm. Super. Pisa Cl. Sci. (4) 19:4, 1992, 591-613.

[20] Kilpeläinen, T., and J. MalÝ: The Wiener test and potential estimates for quasilinear elliptic equations. - Acta Math. 172, 1994, 137-161.

[21] Kilpeläinen, T., and X. Zhong: Growth of entire $\mathcal{A}$-subharmonic functions. - Ann. Acad. Sci. Fenn. Math. 28:1, 2003, 181-192.

[22] Kinderlehrer, D., and G. Stampacchia: An introduction to variational inequalities and their applications. - Pure Appl. Math. 88, Academic Press, New York-London, 1980.

[23] Kinnunen, J., and P. Lindqvist: Pointwise behaviour of semicontinuous supersolutions to a quasilinear parabolic equation. - Ann. Mat. Pura Appl. (4) 185:3, 2006, 411-435.

[24] Lieberman, G.: Second order parabolic differential equations. - World Scientific, Singapore, 1996.

[25] Lindqvist, P.: On the time derivative in an obstacle problem. - Rev. Mat. Iberoam. (to appear), arXiv:1005.2096.

[26] Lindqvist, P., and M. Parviainen: Irregular time dependent obstacles. - Preprint, arXiv:1011.1901.

[27] Lions, J.: Quelques méthodes de résolution des problèmes aux limites non linéaires. - GauthierVillars, Paris, 1969.

[28] Mingione, G.: Gradient potential estimates. - J. Eur. Math. Soc. 13, 2011, 459-486.

[29] Scheven, C.: Nonlinear Calderón-Zygmund theory for parabolic systems with subquadratic growth. - J. Evol. Equ. 10:3, 2010, 597-622.

[30] Scheven, C.: Existence and gradient estimates in nonlinear problems with irregular obstacles. - Habilitation thesis, 2011.

[31] Scheven, C.: Existence of localizable solutions to nonlinear parabolic problems with irregular obstacles. - Preprint, 2011.

[32] Scheven, C.: Elliptic obstacle problems with measure data: Potentials and low order regularity. - Publ. Mat. (to appear).

[33] Scheven, C.: Gradient potential estimates in nonlinear elliptic obstacle problems with measure data. - J. Funct. Anal. 262:6, 2012, 2777-2832.

[34] Showalter, R.: Monotone operators in Banach space and nonlinear partial differential equations. - Amer. Math. Soc., Providence, RI, 1997.

[35] Stein, E., and G. Weiss: Introduction to Fourier analysis on Euclidean spaces. - Princeton Univ. Press, Princeton, N.J., 1971.

[36] Trudinger, N., and X. WANG: On the weak continuity of elliptic operators and applications to potential theory. - Amer. J. Math. 124:2, 2002, 369-410.

[37] Ziemer, W.: Weakly differentiable functions. Sobolev spaces and functions of bounded variation. - Grad. Texts in Math. 120, Springer, New York, 1989.

Received 19 August 2011

Revised received 29 January 2012 